\title{
MAXIMALITY OF BI-INTUITIONISTIC PROPOSITIONAL LOGIC
}

\author{
GRIGORY OLKHOVIKOV AND GUILLERMO BADIA
}

\begin{abstract}
In the style of Lindström's theorem for classical first-order logic, this article characterizes propositional bi-intuitionistic logic as the maximal (with respect to expressive power) abstract logic satisfying a certain form of compactness, the Tarski union property and preservation under bi-asimulations. Since bi-intuitionistic logic introduces new complexities in the intuitionistic setting by adding the analogue of a backwards looking modality, the present paper constitutes a non-trivial modification of previous work done by the authors for intuitionistic $\operatorname{logic}[3]$.

Keywords: Lindström theorem, bi-intuitionistic logic, abstract model theory, bi-asimulations.

Math subject classification: 03C95, 03B55.
\end{abstract}

\section{INTRODUCTION}

In a series of papers from the 1970s, Cecylia Rauszer [27, 28, 29] studied an extension of intuitionistic logic obtained by adding the algebraic dual of the intuitionistic implication $\rightarrow$ to the language. This co-implication connective, which we denote in this paper by "《", is sometimes also called subtraction [26]. In the Kripke semantics for bi-intuitionistic logic, it behaves similarly to a backwards looking diamond modality $\diamond^{-1}$. The resulting logic is known as Heyting-Brouwer or Bi-intuitionistic logic. In recent years, the study of this very natural logic has received some degree of attention from different scholars [1, 2, 13, 14, 15, 21, 23, 24, 25. Our own work in this field has focused on the semantic study of bi-intuitionistic logic. The present contribution continues to develop this line of research, in particular, by studying a Lindström theorem for this logic.

Per Lindström famously provided a battery of results [16, 17, 18, 19, which characterized classical first-order logic as the maximal "abstract logic" (or "model-theoretic language" [10]) satisfying certain combinations of properties from the following list: Compactness, the Löwenheim-Skolem property, the Robinson consistency property, the Karp property, the Tarski Union property, the abstract Completeness property and the $\lambda$-omitting types property. This opened up the possibility of studying logics (involving cardinality quantifiers, infinitary disjunctions and conjunctions, second order quantification, etc.) in accord with what their model theory could offer and situating them in a much broader abstract model-theoretic framework. The textbook references for this subject (called "abstract model theory") are [4, 5].

Lindström theorems have also been studied in the context of non-classical logics [12, with modal logic providing perhaps the most fruitful case [22, 30, 8, 9]. In the context of propositional intuitionistic logic, the authors of the present paper obtained such a theorem in [3] using the Tarski union property, a form of compactness and preservation 
under asimulations (inspired by work done for modal logic in [9]). Since bi-intuitionistic logic is such a natural companion to intuitionistic logic, the question immediately arose whether a similar theorem would hold for bi-intuitionistic logic replacing asimulations (introduced in 20]) by an appropriate bi-intuitionistic analogue (such as that in [3]).

In this article we will adapt the somewhat delicate arguments from 3 to a biintuitionistic context. One of the most substantial difficulties of this task is the backwards looking modality, making the unraveling construction, which plays a central role in our proof, more complex. Hence, to use it the way we need it requires new special care1 Finally, the paper is self-contained and it will not require any familiarity with [3] on behalf of the reader (which, of course, means that we will repeat even the parts from [3] which are rather easily adaptable, and not just the non-trivial parts).

The article is arranged as follows: in $\$ 2$ we establish the main bits of our notation and define some basic concepts. $\S 3$ is then devoted to proving some less immediate lemmas on bi-intuitionistic unravellings and bi-intuitionistically saturated models. In $\$ 4$ we define the notion of an abstract logic in the context of extensions of bi-intuitionistic logic and formulate our main result. In $\$ 5$ we provide the proof of said result. We end the paper by summarizing our work and suggesting some lines of further inquiry.

\section{Preliminaries}

We start this section by fixing some notational conventions to be used in this paper. For any $n>0$, we will denote by $\bar{o}_{n}$ the sequence $\left(o_{1}, \ldots, o_{n}\right)$ of objects of any kind; moreover, somewhat abusing the notation, we will sometimes denote $\left\{o_{1}, \ldots, o_{n}\right\}$ by $\left\{\bar{o}_{n}\right\}$. We will denote by $\left(\bar{o}_{n}\right) \frown\left(\bar{r}_{m}\right)$ the concatenation of $\bar{o}_{n}$ and $\bar{r}_{m}$. The ordered 1-tuple will be identified with its only member, the ordered 0 -tuple will be denoted by $\Lambda$, and the last element of a sequence $\alpha$ will be denoted by end $(\alpha)$ so that we will have, for example, that $\operatorname{end}\left(\bar{o}_{n}\right)=o_{n}$.

If $f$ is any function, then we will denote by $\operatorname{dom}(f)$ its domain and by $\operatorname{rang}(f)$ the image of $\operatorname{dom}(f)$ under $f$; if $\operatorname{rang}(f) \subseteq M$, we will also write $f: \operatorname{dom}(f) \rightarrow M$. Finally, if $X, Y$ are sets, then we will write $X \Subset Y$, if $X \subseteq Y$ and $X$ is finite.

In this paper, we consider the language of bi-intuitionistic propositional logic, which we identify with its set of formulas. This language is generated from some set of propositional letters by a finite number of applications of connectives from the set $\{\perp, \wedge, \vee, \rightarrow, \ll\}$; in this set, « stands for bi-intuitionistic co-implication and the connectives are assumed to have their usual arities. The set of propositional letters can be in general arbitrarily large, but we assume that it is disjoint from the above set of connectives (and from the set of logical symbols of every logic which we are going to consider below). Any set with this property we will call signature. Bi-intuitionistic propositional formulas will be denoted with Greek letters like $\varphi, \psi$ and $\theta[2$ whereas the elements of signatures will be denoted by letters $p, q$, and $r$. If $\Theta$ is a signature, then $B I L(\Theta)$ denotes the set of those bi-intuitionistic propositional formulas which only contain propositional letters from $\Theta$.

\footnotetext{
${ }^{1}$ For instance, the theory encoding all the types of a given model, which is often a pivotal piece in the proofs of this kind, can no longer be given by a finite number of formula schemes; we had to use an inductive definition instead.

${ }^{2}$ We will be adjoining to them subscripts and superscripts when needed, and the same is assumed for any other notations introduced in this paper.
} 
For this language, we assume the standard Kripke semantics. The typical notations for bi-intuitionistic Kripke models that we are going to use below are as follows:

$$
\begin{gathered}
\mathcal{M}=\langle W, \prec, V\rangle, \mathcal{M}^{\prime}=\left\langle W^{\prime}, \prec^{\prime}, V^{\prime}\right\rangle, \mathcal{M}_{n}=\left\langle W_{n}, \prec_{n}, V_{n}\right\rangle, \\
\mathcal{N}=\langle U, \triangleleft, Y\rangle, \mathcal{N}^{\prime}=\left\langle U^{\prime}, \triangleleft^{\prime}, Y^{\prime}\right\rangle, \mathcal{N}_{n}=\left\langle U_{n}, \triangleleft_{n}, Y_{n}\right\rangle,
\end{gathered}
$$

where $n \in \omega$. As we proceed, we will also define some operations on bi-intuitionistic Kripke models which are going to affect the notation.

If $\Theta$ is a signature and $\mathcal{M}$ is a bi-intuitionistic Kripke $\Theta$-model 3 then $W$ is a nonempty set of worlds, states, or nodes, $\prec$ is a partial order on $W$ called $\mathcal{M}$ 's accessibility relation, and $V$ is the evaluation function for $\Theta$ in $\mathcal{M}$, that is to say, a function $V: \Theta \rightarrow 2^{W}$ such that for every $p \in \Theta$ and arbitrary $s, t \in W$, it is true that:

$$
w \prec v \Rightarrow(w \in V(p) \Rightarrow v \in V(p)) .
$$

As usual, we denote the reduct of a $\Theta$-model $\mathcal{M}$ to a smaller signature $\Sigma \subseteq \Theta$ by $\mathcal{M}\lceil\Sigma$. Next, we need a definition of isomorphism between models:

Definition 1. Let $\mathcal{M}, \mathcal{N}$ be $\Theta$-models. A function $g: W \rightarrow U$ is called an isomorphism from $\mathcal{M}$ onto $\mathcal{N}$ (write $g: \mathcal{M} \cong \mathcal{N}$ ) iff $g$ is a bijection, and for all $v, u \in W$ and $p \in \Theta$ it is true that:

$$
\begin{aligned}
& v \prec u \Leftrightarrow g(v) \triangleleft g(u) \\
& v \in V(p) \Leftrightarrow g(v) \in Y(p)
\end{aligned}
$$

If $\mathcal{M}$ and $\mathcal{N}$ are two bi-intuitionistic Kripke $\Theta$-models then we say that $\mathcal{M}$ is a submodel of $\mathcal{N}$ and write $\mathcal{M} \subseteq \mathcal{N}$ iff $W \subseteq U, R=S \uparrow(W \times W)$ and, for every $p \in \Theta$, $V(p)=Y(p) \cap W$. In general, for every $W \subseteq U$ there exists a corresponding submodel $\mathcal{M}$ of $\mathcal{N}$ with $W$ as its universe. Moreover, such an $\mathcal{M}$ is unique up to an isomorphism. In such cases we may also denote $\mathcal{M}$ by $\mathcal{N}(W)$.

If $\mathcal{M}_{1} \subseteq, \ldots, \subseteq \mathcal{M}_{n} \subseteq, \ldots$ is a countable chain of bi-intuitionistic Kripke models then the model:

$$
\bigcup_{n \in \omega} \mathcal{M}_{n}=\left(\bigcup_{n \in \omega} W_{n}, \bigcup_{n \in \omega} \prec_{n}, \bigcup_{n \in \omega} V_{n}\right)
$$

is again a bi-intuitionistic Kripke model.

A pointed bi-intuitionistic Kripke $\Theta$-model is a pair of the form $(\mathcal{M}, w)$ such that $w \in W$. The class of all pointed bi-intuitionistic Kripke models in all signatures (resp. in the signature $\Theta$ ) will be denoted by $P \bmod \left(\right.$ resp. by $\left.P \bmod _{\Theta}\right)$.

In this paper, we are not going to consider any non-bi-intuitionistic Kripke models. Therefore, we will omit the qualification 'bi-intuitionistic Kripke' in what follows and will simply speak about (pointed) $\Theta$-models. We assume the standard satisfaction

\footnotetext{
${ }^{3} \mathrm{Bi}$-intuitionistic Kripke models, as they are defined in this paper, are the same as Kripke models used for intuitionistic propositional logic. Therefore, one can also call them intuitionistic Kripke models. The reason for our preferred terminology is that in this paper we only consider these models in connection with bi-intuitionistic logic and its extensions.
} 
relation for $B I L(\Theta)$ :

$$
\begin{aligned}
& \mathcal{M}, w \models_{B I L} p \Leftrightarrow w \in V(p), \\
& \mathcal{M}, w \models_{B I L} \varphi \wedge \psi \Leftrightarrow \mathcal{M}, w \models_{B I L} \varphi \text { and } \mathcal{M}, w \models_{B I L} \psi ; \\
& \mathcal{M}, w \models_{B I L} \varphi \vee \psi \Leftrightarrow \mathcal{M}, w \models_{B I L} \varphi \text { or } \mathcal{M}, w \models_{B I L} \psi ; \\
& \mathcal{M}, w \models_{B I L} \varphi \rightarrow \psi \Leftrightarrow(\forall v \succ w)\left(\mathcal{M}, v \models_{B I L} \varphi \text { or } \mathcal{M}, v \models_{B I L} \psi\right) ; \\
& \mathcal{M}, w \models_{B I L} \varphi \ll \psi \Leftrightarrow(\exists v \prec w)\left(\mathcal{M}, v \models_{B I L} \varphi \text { and } \mathcal{M}, v \not \models_{B I L} \psi\right) ; \\
& \mathcal{M}, w \models_{B I L} \perp .
\end{aligned}
$$

Note that the formulas of $B I L(\Theta)$ get satisfied at pointed $\Theta$-models rather than at models alone.

Since classical negation is not available in bi-intuitionistic logic, it makes sense to define bi-intuitionistic theories, in the same way as intuitionistic theories are often defined, that is to say as pairs of sets of formulas including truth assumptions and falsehood assumptions of a given theory (see e.g. [11, p. 110] w.r.t. intuitionistic firstorder logic). Thus a $B I L(\Theta)$-theory becomes a pair $(\Gamma, \Delta) \in 2^{B I L(\Theta)} \times 2^{B I L(\Theta)}$, where formulas in $\Gamma$ are assumed to be true and formulas from $\Delta$ are assumed to be false. If $(\mathcal{M}, w)$ is a pointed $\Theta$-model, then we define $T h_{B I L}(\mathcal{M}, w)$, the $B I L(\Theta)$-theory of $(\mathcal{M}, w)$, as follows:

$$
T h_{B I L}(\mathcal{M}, w):=\left(\left\{\varphi \in B I L(\Theta) \mid \mathcal{M}, w \models_{B I L} \varphi\right\},\left\{\varphi \in B I L(\Theta) \mid \mathcal{M}, w \nvdash_{B I L} \varphi\right\}\right) .
$$

We also introduce a special notation for the left and right projection of $T h_{B I L}(\mathcal{M}, w)$, that is to say, for the positive and for the negative part of this theory, denoting them by $T h_{B I L}^{+}(\mathcal{M}, w)$ and $T h_{B I L}^{-}(\mathcal{M}, w)$, respectively. Inclusion of intuitionistic theories must then involve set-theoretic inclusion of their respective projections, so that we define:

$$
(\Gamma, \Delta) \subseteq\left(\Gamma^{\prime}, \Delta^{\prime}\right) \Leftrightarrow \Gamma \subseteq \Gamma^{\prime} \text { and } \Delta \subseteq \Delta^{\prime}
$$

Also the set theoretic operations on the pairs of sets will be understood componentwise, e.g. we will assume that $(\Gamma, \Delta) \cap\left(\Gamma^{\prime}, \Delta^{\prime}\right)=\left(\Gamma \cap \Gamma^{\prime}, \Delta \cap \Delta^{\prime}\right)$ and similarly for $\cup$.

It is clear then that a $B I L(\Theta)$-theory $(\Gamma, \Delta)$ is $B I L$-satisfiable iff we have

$$
(\Gamma, \Delta) \subseteq T h_{B I L}(\mathcal{M}, w)
$$

for some $(\mathcal{M}, w) \in \operatorname{Pmod}_{\Theta}$. In this case we will also write $\mathcal{M}, w \models_{B I L}(\Gamma, \Delta)$. If $\mathcal{M} \subseteq \mathcal{N}$ and for every $w \in W$ it is true that $T h_{B I L}(\mathcal{M}, w)=T h_{B I L}(\mathcal{N}, w)$, then we say that $\mathcal{M}$ is an $B I L$-elementary submodel of $\mathcal{N}$ and write $\mathcal{M} \preccurlyeq_{B I L} \mathcal{N}$. A standard generalization of the notion of a $B I L$-elementary submodel leads as to the concept of $B I L$-elementary embedding:

Definition 2. Let $\mathcal{M}, \mathcal{N}$ be $\Theta$-models. An injective function $g: W \rightarrow U$ is called a $B I L$-elementary embedding of $\mathcal{M}$ into $\mathcal{N}$ iff for all $v, u \in W$ it is true that

$$
\begin{aligned}
& v \prec u \Leftrightarrow g(v) \triangleleft g(u) \\
& T h_{B I L}(\mathcal{M}, v)=T h_{B I L}(\mathcal{N}, g(v))
\end{aligned}
$$

We collect some of the easy consequences of our series of definitions in the following lemma:

Lemma 1. Let $\mathcal{M}, \mathcal{N}$ be $\Theta$-models and let $\Sigma \subseteq \Theta$. Then the following statements hold:

(1) $T h_{B I L}(\mathcal{M}, w) \cap(B I L(\Sigma), B I L(\Sigma))=T h_{B I L}(\mathcal{M}\lceil\Sigma, w)$. 
(2) If $\mathcal{M} \preccurlyeq_{B I L} \mathcal{N}$, then $\left(\mathcal{M}\lceil\Sigma) \preccurlyeq{ }_{B I L}(\mathcal{N}\lceil\Sigma)\right.$.

(3) If $\mathcal{M} \preccurlyeq_{B I L} \mathcal{N}$, then the identical function $i d_{W}$ is a BIL-elementary embedding of $\mathcal{M}$ into $\mathcal{N}$.

(4) If $g: \mathcal{M} \cong \mathcal{N}$, then $g$ is a BIL-elementary embedding of $\mathcal{M}$ into $\mathcal{N}$; in particular, for any $w \in W$, we have $T h_{B I L}(\mathcal{M}, w)=T h_{B I L}(\mathcal{N}, g(w))$.

(5) A function $g$ is a BIL-elementary embedding of $\mathcal{M}$ into $\mathcal{N}$ iff there exists a (unique) $\mathcal{N}^{\prime} \preccurlyeq B I L \mathcal{N}$ such that $g: \mathcal{M} \cong \mathcal{N}^{\prime}$. We will denote $\mathcal{N}^{\prime}$ by $g(\mathcal{M})$.

(6) If $g$ is a BIL-elementary embedding of $\mathcal{M}$ into $\mathcal{N}$, then there exists a $\Theta$-model $\mathcal{M}^{\prime}$ and function $g^{\prime}$ such that we have: (a) $\mathcal{M} \preccurlyeq B I L \mathcal{M}^{\prime}$, (b) $g \subseteq g^{\prime}$, and (c) $g^{\prime}: \mathcal{M}^{\prime} \cong \mathcal{N}$.

Proof. Part 1 is immediate from the definition, as for Part 2, it is clear that if $\mathcal{M} \subseteq \mathcal{N}$, then also $(\mathcal{M}\lceil\Sigma) \subseteq(\mathcal{N}\lceil\Sigma)$. It remains to show the coincidence of theories in the reducts of $\mathcal{M}$ and $\mathcal{N}$. We reason as follows for any given $w \in W$ :

$$
\begin{aligned}
T h_{B I L}(\mathcal{M}\lceil\Sigma, w) & =T h_{B I L}(\mathcal{M}, w) \cap(B I L(\Sigma), B I L(\Sigma)) & & \text { (by Part 1) } \\
& =T h_{B I L}(\mathcal{N}, w) \cap(B I L(\Sigma), B I L(\Sigma)) & & \text { by } \mathcal{M} \preccurlyeq B I L \mathcal{N}) \\
& =T h_{B I L}(\mathcal{N}\lceil\Sigma, w) & & \text { (by Part 1) }
\end{aligned}
$$

Thus we get that $(\mathcal{M}\lceil\Sigma) \preccurlyeq B I L(\mathcal{N}\lceil\Sigma)$.

Parts $3-5$ are straightforward. As for Part 6 , we reason as in the classical case, that is to say, we choose a set $C$ outside $W \cup U$ such that there exists a bijection $g^{\prime \prime}: C \rightarrow(U \backslash h(W))$. We then set $g^{\prime}:=g \cup g^{\prime \prime}$ and define $\mathcal{M}^{\prime}$ on the basis of $W^{\prime}:=W \cup C$, setting $u \prec^{\prime} u^{\prime}: \Leftrightarrow g^{\prime}(u) \triangleleft g^{\prime}\left(u^{\prime}\right)$ and $u \in V^{\prime}(p): \Leftrightarrow g^{\prime}(u) \in Y(p)$ for all $p \in \Theta$ and $u, u^{\prime} \in W^{\prime}$.

We are now in a position to define the notion of a bi-intuitionistic type:

Definition 3. Let $(\mathcal{M}, w) \in \operatorname{Pmod}_{\Theta}$, let $\Gamma, \Delta \subseteq B I L(\Theta)$. Then we say that:

- $(\Gamma, \Delta)$ is a successor BIL-type of $(\mathcal{M}, w)$ iff:

$$
\left(\forall \Gamma^{\prime} \Subset \Gamma\right)\left(\forall \Delta^{\prime} \Subset \Delta\right)(\exists v \succ w)\left(\left(\Gamma^{\prime}, \Delta^{\prime}\right) \subseteq T h_{B I L}(\mathcal{M}, v)\right) .
$$

- $(\Gamma, \Delta)$ is a predecessor BIL-type of $(\mathcal{M}, w)$ iff:

$$
\left(\forall \Gamma^{\prime} \Subset \Gamma\right)\left(\forall \Delta^{\prime} \Subset \Delta\right)(\exists v \prec w)\left(\left(\Gamma^{\prime}, \Delta^{\prime}\right) \subseteq T h_{B I L}(\mathcal{M}, v)\right) .
$$

It is easy to see that, due to the reflexivity of the accessibility relation $T h_{B I L}(\mathcal{M}, w)$ is always both a successor and a predecessor type of $(\mathcal{M}, w)$. More generally, we will say that a pair of sets of formulas is a $B I L$-type of $(\mathcal{M}, w)$ iff it is either a successor or a predecessor type of $(\mathcal{M}, w)$; we will say that it is a $B I L$-type of $\mathcal{M}$ iff it is a type of $(\mathcal{M}, w)$ for some $w \in W$.

Another straightforward observation is that the bi-intuitionistic types, as given by Definition 3. are related to certain classes of bi-intuitionistic formulas. We state this observation as a corollary (omitting the obvious proof):

Corollary 1. Let $(\mathcal{M}, w) \in \operatorname{Pmod}_{\Theta}$, let $\Gamma, \Delta \subseteq B I L(\Theta)$. Then all of the following statements hold:

(1) $(\Gamma, \Delta)$ is a successor BIL-type of $(\mathcal{M}, w)$ iff:

$$
\left(\forall \Gamma^{\prime} \Subset \Gamma\right)\left(\forall \Delta^{\prime} \Subset \Delta\right)\left(\mathcal{M}, w \forall_{B I L} \bigwedge \Gamma^{\prime} \rightarrow \bigvee \Delta^{\prime}\right)
$$


(2) $(\Gamma, \Delta)$ is a predecessor BIL-type of $(\mathcal{M}, w)$ iff:

$$
\left(\forall \Gamma^{\prime} \Subset \Gamma\right)\left(\forall \Delta^{\prime} \Subset \Delta\right)\left(\mathcal{M}, w \models_{B I L} \bigwedge \Gamma^{\prime} \ll \bigvee \Delta^{\prime}\right)
$$

We now define what it means for a bi-intuitionistic type to be realized:

Definition 4. Let $(\mathcal{M}, w) \in \operatorname{Pmod}_{\Theta}$, let $\mathcal{M} \preccurlyeq B I L \mathcal{N}$, let $\Gamma, \Delta \subseteq B I L(\Theta)$. Then we say that:

- If $(\Gamma, \Delta)$ is a successor BIL-type of $(\mathcal{M}, w)$, then $(\Gamma, \Delta)$ is realized in $\mathcal{N}$ iff there exists a $v \in U$ such that $w \triangleleft v$ and we have $(\Gamma, \Delta) \subseteq T h_{B I L}(\mathcal{N}, v)$.

- If $(\Gamma, \Delta)$ is a predecessor BIL-type of $(\mathcal{M}, w)$, then $(\Gamma, \Delta)$ is realized in $\mathcal{N}$ iff there exists a $v \in U$ such that $v \triangleleft w$ and we have $(\Gamma, \Delta) \subseteq T h_{B I L}(\mathcal{N}, v)$.

For an intuitionistic model to realize all of its bi-intuitionistic types in the sense of Definition 4 is a rare property that merits a special name:

Definition 5. Let $\mathcal{M}$ be a $\Theta$-model. We say that $\mathcal{M}$ is BIL-saturated iff it realizes all BIL-types of $\mathcal{M}$.

Another interesting situation occurs when a model happens to realize every type of its proper $B I L$-elementary submodel. We collect some properties of type realization in the following lemma:

Lemma 2. Let $\mathcal{M}^{\prime} \preccurlyeq_{B I L} \mathcal{M} \preccurlyeq_{B I L} \mathcal{N}$ be a BIL-elementary chain of $\Theta$-models, let $\mathcal{N}^{\prime}$ be a $\Theta$-model such that for some functions $g$ we have $g: \mathcal{N} \cong \mathcal{N}^{\prime}$. Finally, let $\Sigma \subseteq \Theta$. Then the following statements hold:

(1) Every BIL-type of $\mathcal{M}^{\prime}$, that is realized in $\mathcal{M}$, is also realized in $\mathcal{N}$.

(2) If $\mathcal{N}$ realizes every BIL-type of $\mathcal{M}$, then $\mathcal{N} \backslash \Sigma$ realizes every BIL-type of $\mathcal{M} \uparrow \Sigma$.

(3) For every $v \in U,(\Gamma, \Delta)$ is a successor (resp. predecessor) BIL-type of $(\mathcal{N}, v)$ iff it is a successor (resp. predecessor) BIL-type of $\left(\mathcal{N}^{\prime}, g(v)\right)$.

Proof. (Part 1). Assume that $(\Gamma, \Delta)$ is a successor BIL-type of $\left(\mathcal{M}^{\prime}, w\right)$ that is realized in $\mathcal{M}$. This means that for an appropriate $v \in W$ such that $w \prec v$ we have $(\Gamma, \Delta) \subseteq T h_{B I L}(\mathcal{M}, v)=T h_{B I L}(\mathcal{N}, v)$. Note that, by $\mathcal{M} \preccurlyeq B I L \mathcal{N}$, we also have $w \triangleleft v$, whence $(\Gamma, \Delta)$ is also realized in $\mathcal{N}$. We argue similarly in case when $(\Gamma, \Delta)$ is a predecessor $B I L$-type.

(Part 2). Assume that $w \in W$, and that $(\Gamma, \Delta)$ is a successor (resp. predecessor) $B I L$-type of $(\mathcal{M} \uparrow \Sigma, w)$. Then it follows from the respective part of Corollary 1 that $(\Gamma, \Delta)$ is also a successor (resp. predecessor) $B I L$-type of $(\mathcal{M}, w)$. But then $(\Gamma, \Delta)$ must be realized in $\mathcal{N}$, which means, by definition, that for an appropriate $v \in U$ such that $w \triangleleft v$ (resp. $v \triangleleft w)$, we have that $(\Gamma, \Delta) \subseteq T h_{B I L}(\mathcal{N}, v)$. But this means, further, that we also have:

$$
\begin{aligned}
(\Gamma, \Delta) & =(\Gamma \cap B I L(\Sigma), \Delta \cap B I L(\Sigma))=(\Gamma, \Delta) \cap B I L(\Sigma) \\
& \subseteq T h_{B I L}(\mathcal{N}, v) \cap(B I L(\Sigma), B I L(\Sigma))=T h_{B I L}(\mathcal{N}\lceil\Sigma, v),
\end{aligned}
$$

where the last equality holds by Lemma 1, 1 . This means that $(\Gamma, \Delta)$ is also realized in $\mathcal{N}\lceil\Sigma$.

(Part 3). By Corollary 1 and Lemma 14. 


\section{Bi-Asimulations And Bi-UnRAVEllings}

We devote this subsection to adaptations of some classic constructions in the model theory of classical modal logic to the case of bi-intuitionistic logic. The first one is the bi-intuitionistic analogue of bisimulation relation, originally introduced in [1]:

Definition 6. Let $\left(\mathcal{M}_{1}, w_{1}\right),\left(\mathcal{M}_{2}, w_{2}\right) \in \operatorname{Pmod}_{\Theta}$. A binary relation $A$ is called a bi-asimulation from $\left(\mathcal{M}_{1}, w_{1}\right)$ to $\left(\mathcal{M}_{2}, w_{2}\right)$ iff for any $i, j$ such that $\{i, j\}=\{1,2\}$, any $v \in W_{i}, s, t \in W_{j}$, any propositional letter $p \in \Theta$ the following conditions hold:

$\begin{array}{ll}\text { (w-type) } & A \subseteq\left(W_{1} \times W_{2}\right) \cup\left(W_{2} \times W_{1}\right) \\ \text { (elem) } & w_{1} A w_{2} \\ \text { (s-atom) } & \left.\left.\left(v A s \wedge v \in V_{i}(p)\right) \Rightarrow s \in V_{j}(p)\right)\right) \\ \text { (s-back) } & \left(v A s \wedge s R_{j} t\right) \Rightarrow \exists u \in W_{i}\left(v R_{i} u \wedge t A u \wedge u A t\right) \\ \text { (s-forth) } & \left(v A s \wedge u R_{i} v\right) \Rightarrow \exists t \in W_{j}\left(t R_{j} s \wedge t A u \wedge u A t\right)\end{array}$

Bi-intuitionistic propositional formulas are known to be preserved under bi-asimulations. More precisely, if $\left(\mathcal{M}_{1}, w_{1}\right)$, and $\left(\mathcal{M}_{2}, w_{2}\right)$ are pointed $\Theta$-models and $A$ is an biasimulation from $\left(\mathcal{M}_{1}, w_{1}\right)$ to $\left(\mathcal{M}_{2}, w_{2}\right)$, then $T h_{B I L}^{+}\left(\mathcal{M}_{1}, w_{1}\right) \subseteq T h_{B I L}^{+}\left(\mathcal{M}_{2}, w_{2}\right)$. Moreover, preservation under bi-asimulations is known to semantically characterize $B I L$ as a fragment of classical first-order logic, see [1, 21 for proofs.

Next we consider bi-asimulations between $B I L$-saturated models. The following lemma states that such bi-asimulations can be defined in an easy and natural way:

Lemma 3. Let $\left(\mathcal{M}_{1}, w_{1}\right),\left(\mathcal{M}_{2}, w_{2}\right) \in \operatorname{Pmod}_{\Theta}$. If

$$
T h_{B I L}^{+}\left(\mathcal{M}_{1}, w_{1}\right) \subseteq T h_{B I L}^{+}\left(\mathcal{M}_{2}, w_{2}\right)
$$

and both $\mathcal{M}_{1}$ and $\mathcal{M}_{2}$ are intuitionistically saturated, then the relation $A$ such that for all $u \in W_{i}, s \in W_{j}$ if $\{i, j\}=\{1,2\}$, then

$$
u A s \Leftrightarrow\left(T h_{B I L}^{+}\left(\mathcal{M}_{i}, u\right) \subseteq T h_{B I L}^{+}\left(\mathcal{M}_{j}, s\right)\right)
$$

is a bi-asimulation from $\left(\mathcal{M}_{1}, w_{1}\right)$ to $\left(\mathcal{M}_{2}, w_{2}\right)$.

Proof. The relation $A$, as defined in the lemma, obviously satisfies conditions w-type, (elem), and (s-atom given in Definition 6. We check the remaining conditions.

Condition (s-back). Assume that $u A s$, so that $T h_{B I L}^{+}\left(\mathcal{M}_{i}, u\right) \subseteq T h_{B I L}^{+}\left(\mathcal{M}_{j}, s\right)$, and that for some $t \in W_{j}$ we have $s R_{j} t$. Let $\left(\Gamma^{\prime}, \Delta^{\prime}\right) \subseteq T h_{B I L}\left(\mathcal{M}_{j}, t\right)$ be finite. Then, of course, we have:

$$
\mathcal{M}_{j}, s \not{ }_{B I L} \bigwedge \Gamma^{\prime} \rightarrow \bigvee \Delta^{\prime}
$$

and, by $u A s$ :

$$
\mathcal{M}_{i}, u \forall \forall_{B I L} \bigwedge \Gamma^{\prime} \rightarrow \bigvee \Delta^{\prime}
$$

The latter means that $\left(\Gamma^{\prime}, \Delta^{\prime}\right)$ must be $B I L$-satisfied by some $R_{i}$-successor of $u$. Therefore, by bi-intuitionistic saturation of both $\mathcal{M}_{1}$ and $\mathcal{M}_{2}$, we get that for some $w \in W_{i}$ such that $u R_{i} w$ the theory $T h_{B I L}\left(\mathcal{M}_{j}, t\right)$ is $B I L$-satisfied at $\left(\mathcal{M}_{i}, w\right)$. It follows immediately that

$$
T h_{B I L}\left(\mathcal{M}_{i}, w\right)=T h_{B I L}\left(\mathcal{M}_{j}, t\right)
$$

whence

$$
T h_{B I L}^{+}\left(\mathcal{M}_{i}, w\right)=T h_{B I L}^{+}\left(\mathcal{M}_{j}, t\right),
$$

which, in turn, means that both $w A t$ and $t A w$. 
Condition (s-forth). Assume that $u A s$, so that $T h_{B I L}^{+}\left(\mathcal{M}_{i}, u\right) \subseteq T h_{B I L}^{+}\left(\mathcal{M}_{j}, s\right)$, and that for some $w \in W_{i}$ we have $w R_{i} u$. Let $\left(\Gamma^{\prime}, \Delta^{\prime}\right) \subseteq T h_{B I L}\left(\mathcal{M}_{i}, w\right)$ be finite. Then, of course, we have:

$$
\mathcal{M}_{i}, u \models_{B I L} \bigwedge \Gamma^{\prime} \ll \bigvee \Delta^{\prime}
$$

and, by $u A s$ :

$$
\mathcal{M}_{j}, s \models_{B I L} \bigwedge \Gamma^{\prime} \ll \bigvee \Delta^{\prime} .
$$

The latter means that $\left(\Gamma^{\prime}, \Delta^{\prime}\right)$ must be $B I L$-satisfied by some $R_{j}$-predecessor of $s$. Therefore, by bi-intuitionistic saturation of both $\mathcal{M}_{1}$ and $\mathcal{M}_{2}$, we get that for some $t \in W_{j}$ such that $t R_{j} s$ the theory $T h_{B I L}\left(\mathcal{M}_{i}, w\right)$ is $B I L$-satisfied at $\left(\mathcal{M}_{j}, t\right)$. It follows immediately that

$$
T h_{B I L}\left(\mathcal{M}_{i}, w\right)=T h_{B I L}\left(\mathcal{M}_{j}, t\right),
$$

whence

$$
T h_{B I L}^{+}\left(\mathcal{M}_{i}, w\right)=T h_{B I L}^{+}\left(\mathcal{M}_{j}, t\right),
$$

which, in turn, means that both $w A t$ and $t A w$.

The second idea is a bi-intuitionistic adaptation of unravelling, a well-known technique also applicable, as seen in [7, to intuitionistic propositional logic. For the setting of $B I L$ we modify this idea to obtain the notion of bi-unravelling.

For a given $(\mathcal{M}, w) \in \operatorname{Pmod}_{\Theta}$, the model $\mathcal{M}_{w}^{u n}=\left\langle W_{w}^{u n}, \prec_{w}^{u n}, V_{w}^{u n}\right\rangle$, called the biunravelling of $\mathcal{M}$ around $w$ is defined as follows.

- $W_{w}^{u n}=\left\{\bar{u}_{n} \in W^{n} \mid u_{1}=w,(\forall i<n)\left(u_{i}(\prec \cup \succ) u_{i+1} \& u_{i} \neq u_{i+1}\right)\right\}$; in other words, $W_{w}^{u n}$ is the set of finite $(\prec \cup \succ)$-chains starting at $w$ where no two adjacent nodes coincide.

- $\prec_{w}^{u n}$ is the reflexive and transitive closure of the following relation:

$$
\begin{array}{r}
\rho_{w}^{u n}=\left\{(s, t) \in W_{w}^{u n} \times W_{w}^{u n} \mid\left(\exists n<\omega \exists \bar{w}_{n+1} \in W^{n+1}\right)\left(\left(s=\bar{w}_{n} \& t=\bar{w}_{n+1} \& w_{n} \prec w_{n+1}\right) \vee\right.\right. \\
\left.\left.\left(s=\bar{w}_{n+1} \& t=\bar{w}_{n} \& w_{n} \succ w_{n+1}\right)\right)\right\} ;
\end{array}
$$

- For arbitrary $p \in \Theta$, we have $V_{w}^{u n}(p)=\left\{\bar{u}_{n} \in W_{w}^{u n} \mid u_{n} \in V(p)\right\}$.

The structure of bi-unravellings is a bit more complicated than the structure of simple unravellings applied in classical modal logic and intuitionistic logic. In particular, bi-unravellings do not typically lead to tree-like frames. Therefore, before we relate biunravellings to bi-asimulations and bi-intuitionistic theories, we need to look somewhat more closely into the structure of bi-unravelled models. We do this by proving a couple of technical lemmas.

Lemma 4. Let $(\mathcal{M}, w) \in \operatorname{Pmod}_{\Theta}$, let $k>0$, let the tuple $\bar{\alpha}_{k} \in\left(W_{w}^{u n}\right)^{k}$ consist of pairwise distinct tuples such that for all $i<k$ we have $\alpha_{i} \rho_{w}^{u n} \alpha_{i+1}$. Then there exist $m, n \geq 0$ and (not necessarily pairwise distinct) $\bar{v}_{m} \in W^{m}$ and $\bar{u}_{n} \in W^{n}$ such that: (1) $v_{m} \prec \ldots \prec v_{1} \prec \operatorname{end}\left(\alpha_{m+1}\right) \prec u_{1} \prec \ldots \prec u_{n}$, (2) $k=m+n+1$, (3) for all $1 \leq i \leq m, \alpha_{i}=\left(\alpha_{m+1}\right)^{-} \bar{v}_{m-i+1}$, and (4) for all $1 \leq i \leq n, \alpha_{m+1+i}=\left(\alpha_{m+1}\right)^{\frown} \bar{u}_{i}$. 
In other words, $\bar{\alpha}_{k}$ is representable in the form:

$$
\begin{gathered}
\alpha_{1}=\left(\alpha_{m+1}\right) \frown\left(\bar{v}_{m}\right) \\
\vdots \\
\alpha_{m}=\left(\alpha_{m+1}\right) \frown v_{1} \\
\alpha_{m+1} \\
\alpha_{m+2}=\left(\alpha_{m+1}\right) \frown u_{1} \\
\vdots \\
\alpha_{k}=\alpha_{m+n+1}=\left(\alpha_{m+1}\right) \frown\left(\bar{u}_{n}\right) .
\end{gathered}
$$

Proof. We proceed by induction on $k>0$.

Induction Basis. $k=1$. Then we set $m=n:=0, \bar{v}_{m}=\bar{u}_{n}:=\Lambda$, and get the Lemma trivially satisfied.

Induction Step. $k=l+1$ for some $l \geq 1$. Then compare $\alpha_{1}$ and $\alpha_{2}$. Two cases are possible:

Case 1. There exist $\bar{w}_{r+1} \in W^{r+1}$ such that $\alpha_{1}=\bar{w}_{r+1}, \alpha_{2}=\bar{w}_{r}$, and $w_{r} \succ w_{r+1}$. Then we apply Induction Hypothesis to $\left(\alpha_{2}, \ldots, \alpha_{k}\right)$ and find $m_{1}, n_{1} \geq 0$ and (not necessarily pairwise distinct) $\bar{v}_{m_{1}}^{\prime} \in W^{m_{1}}$ and $\bar{u}_{n_{1}}^{\prime} \in W^{n_{1}}$ such that: (1) $v_{m_{1}}^{\prime} \prec \ldots \prec v_{1}^{\prime} \prec \operatorname{end}\left(\alpha_{m_{1}^{\prime}+2}\right) \prec u_{1}^{\prime} \prec \ldots \prec u_{n_{1}}^{\prime}$, (2) $k-1=m_{1}+n_{1}+1$, (3) for all $1 \leq i \leq m_{1}, \alpha_{i+1}=\left(\alpha_{m_{1}+2}\right)^{-} \bar{v}_{m_{1}-i+1}^{\prime}$, and (4) for all $1 \leq i \leq n_{1}, \alpha_{m_{1}+2+i}=\left(\alpha_{m_{1}+2}\right) \frown \bar{u}_{i}^{\prime}$. In other words, $\left(\alpha_{2}, \ldots, \alpha_{k}\right)$ is representable in the form:

$$
\begin{gathered}
\alpha_{2}=\left(\alpha_{m_{1}+2}\right) \frown \bar{v}_{m_{1}}^{\prime} \\
\vdots \\
\alpha_{m_{1}+1}=\left(\alpha_{m_{1}+2}\right) \frown v_{1}^{\prime} \\
\alpha_{m_{1}+2} \\
\alpha_{m_{1}+2}=\left(\alpha_{m_{1}+2}\right) \frown u_{1}^{\prime} \\
\vdots \\
\alpha_{k}=\alpha_{m_{1}+n_{1}+2}=\left(\alpha_{m_{1}+2}\right) \frown \bar{u}_{n_{1}}^{\prime}
\end{gathered}
$$

But then we can set $m:=m_{1}+1, n:=n_{1}, \bar{v}_{m}:=\left(\bar{v}_{m_{1}}^{\prime}\right) \frown\left(w_{r+1}\right), \bar{u}_{n}:=\bar{u}_{n_{1}}^{\prime}$, and get the Lemma verified.

Case 2. There exist $\bar{w}_{r+1} \in W^{r+1}$ such that $\alpha_{1}=\bar{w}_{r}, \alpha_{2}=\bar{w}_{r+1}$, and $w_{r} \prec w_{r+1}$. Again we apply Induction Hypothesis to $\left(\alpha_{2}, \ldots, \alpha_{k}\right)$ and find $m_{1}, n_{1} \geq 0$ and (not necessarily pairwise distinct) $\bar{v}_{m_{1}}^{\prime} \in W^{m_{1}}$ and $\bar{u}_{n_{1}}^{\prime} \in W^{n_{1}}$ allowing to represent $\left(\alpha_{2}, \ldots, \alpha_{k}\right)$ in the form given in Case 1. But then, we must have $\left(\alpha_{m_{1}+2}\right)^{\frown} \bar{v}_{m_{1}}^{\prime}=\alpha_{2}=\bar{w}_{r+1}$. Now, assume that $m_{1}>0$; then we would have $v_{m_{1}}^{\prime}=w_{r+1}$, and also $\alpha_{3}=\left(\alpha_{m_{1}+2}\right)^{-} \bar{v}_{m_{1}-1}^{\prime}=\bar{w}_{r}=\alpha_{1}$, but the latter is in contradiction with our assumption that $\bar{\alpha}_{k} \in\left(W_{w}^{u n}\right)^{k}$ consists of pairwise distinct tuples. Therefore, we must have $m_{1}=0$, so that $\left(\alpha_{2}, \ldots, \alpha_{k}\right)$ in fact 
must have the form:

$$
\begin{aligned}
\alpha_{2} & =\alpha_{m_{1}+2}=\bar{w}_{r+1} \\
\alpha_{3} & =\alpha_{m_{1}+2}=\left(\alpha_{m_{1}+2}\right) \frown u_{1}^{\prime} \\
& \vdots \\
\alpha_{k} & =\alpha_{m_{1}+n_{1}+2}=\left(\alpha_{m_{1}+2}\right) \frown \bar{u}_{n_{1}}^{\prime}
\end{aligned}
$$

But then we can set $m:=0, n:=n_{1}+1, \bar{v}_{m}:=\Lambda, \bar{u}_{n}:=\left(w_{r+1}\right) \frown \bar{u}_{n_{1}}^{\prime}$; these settings clearly satisfy the Lemma.

Lemma 5. Let $(\mathcal{M}, w) \in \operatorname{Pmod}_{\Theta}$, let $\alpha, \beta \in\left(W_{w}^{u n}\right)$ be such that $\alpha \prec_{w}^{u n} \beta$. Then there exist $\gamma \in W_{w}^{u n}, m, n \geq 0$ and (not necessarily pairwise distinct) $\bar{v}_{m} \in W^{m}$ and $\bar{u}_{n} \in W^{n}$ such that $v_{m} \prec \ldots \prec v_{1} \prec \operatorname{end}(\gamma) \prec u_{1} \prec \ldots \prec u_{n}$, and:

$$
\begin{aligned}
& \alpha=\gamma \frown \bar{v}_{m} \\
& \beta=\gamma \succ \bar{u}_{n} .
\end{aligned}
$$

Proof. Since $\prec_{w}^{u n}$ is just the reflexive and transitive closure of $\rho_{w}^{u n}$, there must be an $l \geq 1$ and $\bar{\alpha}_{l}^{\prime} \in\left(W_{w}^{u n}\right)^{l}$ such that $\alpha_{1}^{\prime} \rho_{w}^{u n} \ldots \rho_{w}^{u n} \alpha_{l}^{\prime}, \alpha=\alpha_{1}^{\prime}$ and $\beta=\alpha_{l}^{\prime}$. In case $\bar{\alpha}_{l}$ consists of tuples that are not pairwise distinct, we can just replace in $\bar{\alpha}_{l}^{\prime}$ every sub-sequence of the form $\beta \frown\left(\bar{\delta}_{r}\right) \frown \beta$ with $\beta$. The result of these replacements will be some $\bar{\alpha}_{k} \in\left(W_{w}^{u n}\right)^{k}$ consisting of pairwise distinct tuples, such that $k>0$, $\alpha_{1} \rho_{w}^{u n} \ldots \rho_{w}^{u n} \alpha_{k}, \alpha=\alpha_{1}$ and $\beta=\alpha_{k}$. But then we can apply Lemma 4. to find $m, n \geq 0$ and (not necessarily pairwise distinct) $\bar{v}_{m} \in W^{m}$ and $\bar{u}_{n} \in W^{n}$ such that $v_{m} \prec \ldots \prec v_{1} \prec \operatorname{end}\left(\alpha_{m+1}\right) \prec u_{1} \prec \ldots \prec u_{n}$, and we have:

$$
\begin{gathered}
\alpha_{1}=\left(\alpha_{m+1}\right) \frown\left(\bar{v}_{m}\right) \\
\vdots \\
\alpha_{m}=\left(\alpha_{m+1}\right) \frown v_{1} \\
\alpha_{m+1} \\
\alpha_{m+2}=\left(\alpha_{m+1}\right) \frown u_{1} \\
\vdots \\
\alpha_{k}=\alpha_{m+n+1}=\left(\alpha_{m+1}\right) \frown\left(\bar{u}_{n}\right) .
\end{gathered}
$$

Setting then $\gamma:=\alpha_{m+1}$, we get our Lemma verified.

The following two lemmas sum up the basic facts about bi-unravellings:

Lemma 6. Let $(\mathcal{M}, w) \in \operatorname{Pmod}_{\Theta}$. Then $\left(\mathcal{M}_{w}^{u n}, w\right) \in \operatorname{Pmod}_{\Theta}$.

Proof. By definition, $\prec_{w}^{u n}$ is reflexive and transitive. As for antisymmetry, assume that, for a given $\bar{w}_{r}, \bar{v}_{n} \in W_{w}^{u n}$, we have

$$
\bar{w}_{r} \prec_{w}^{u n} \bar{v}_{n} \& \bar{v}_{n} \prec_{w}^{u n} \bar{w}_{r} .
$$

By Lemma 5, there must be, on the one hand, some $m_{0}, n_{0} \geq 0$ and $\gamma \in W_{w}^{u n}$ and (not necessarily pairwise distinct) $\bar{v}_{m_{0}} \in W^{m_{0}}$ and $\bar{u}_{n_{0}} \in W^{n_{0}}$ such that $v_{m_{0}} \prec \ldots \prec v_{1} \prec u_{1} \prec \ldots \prec u_{n_{0}}$, 
and:

$$
\begin{aligned}
& \alpha=\gamma^{\complement} \bar{v}_{m_{0}} \\
& \beta=\gamma^{\frown} \bar{u}_{n_{0}} .
\end{aligned}
$$

On the other hand, again by Lemma 5 , there must be some $m_{1}, n_{1} \geq 0$ and $\delta \in W_{w}^{u n}$ and (not necessarily pairwise distinct) $\bar{s}_{m_{1}} \in W^{m_{1}}$ and $\bar{t}_{n_{1}} \in W^{n_{1}}$ such that $s_{m_{1}} \prec \ldots \prec s_{1} \prec t_{1} \prec \ldots \prec t_{n_{1}}$, and:

$$
\begin{aligned}
& \beta=\delta^{\frown} \bar{s}_{m_{1}} \\
& \alpha=\delta^{\frown} \bar{t}_{n_{1}} .
\end{aligned}
$$

We then compare $l_{\gamma}$ and $l_{\delta}$, the lengths of $\gamma$ and $\delta$, respectively. Assume, w.l.o.g., that $l_{\gamma} \geq l_{\delta}$. We must have then $\gamma \neg \bar{v}_{m_{0}}=\alpha=\delta \frown \bar{t}_{n_{1}}$, whence it follows that $\gamma=\delta \frown \bar{t}_{n_{1}-m_{0}}$ and so also $\bar{v}_{m_{0}}=\left(t_{n_{1}-m_{0}+1}, \ldots, t_{n_{1}}\right)$. But then, notice that we have both $v_{m_{0}} \prec \ldots \prec v_{1}$ and $t_{n_{1}-m_{0}+1} \prec \ldots \prec t_{n_{1}}$. It follows then, by the antisymmetry of $\prec$, that we have:

$$
v_{m_{0}}=t_{n_{1}-m_{0}+1}=\ldots=v_{1}=t_{n_{1}}
$$

so, in particular, we get that $v_{1}=\ldots=v_{m_{0}}$. Next, note we have $\alpha=\gamma \frown \bar{v}_{m_{0}} \in W_{w}^{u n}$, which means that no two adjacent elements in $\bar{v}_{m_{0}}$ may coincide. Hence we must have $m_{0}=1$, and thus also $\alpha=\gamma \frown v_{1}$.

By a parallel argument, we get that, on the other hand, we must have $\gamma=\delta^{\frown} \bar{s}_{m_{1}-n_{0}}$ and also $\bar{u}_{n_{0}}=\left(s_{m_{1}-n_{0}+1}, \ldots, s_{m_{1}}\right)$. But then, notice that we have both $u_{1} \prec \ldots \prec u_{n_{0}}$ and $s_{m_{1}} \prec \ldots \prec s_{m_{1}-n_{0}+1}$, whence it follows, by the antisymmetry of $\prec$, that we have:

$$
u_{1}=s_{m_{1}}=\ldots=u_{n_{0}}=s_{m_{1}-n_{0}+1}
$$

so, in particular, we get that $u_{1}=\ldots=u_{n_{0}}$. Next, note we have $\beta=\gamma \frown \bar{u}_{n_{0}} \in W_{w}^{u n}$, which means that no two adjacent elements in $\bar{v}_{m_{0}}$ may coincide. Hence we must have $n_{0}=1$, and thus also $\beta=\gamma \frown u_{1}$.

Furthermore, note that, by the choice of $v_{1}$ and $u_{1}$, we have $v_{1} \prec u_{1}$. On the other hand, the above chains of equalities, together with the transitivity of $\prec$, also imply that $u_{1}=s_{m_{1}-n_{0}+1} \prec t_{n_{1}}=v_{1}$, hence also $u_{1} \prec v_{1}$. It follows, next, by the antisymmetry of $\prec$, that $u_{1}=v_{1}$, so that we get that:

$$
\alpha=\gamma \frown v_{1}=\gamma \frown u_{1}=\beta .
$$

To show monotonicity of $V_{w}^{u n}$ w.r.t. $\prec_{w}^{u n}$, assume that for some $\alpha, \beta \in W_{w}^{u n}$ we have $\alpha \prec_{w}^{u n} \beta$. Then we choose, using Lemma 5, $\gamma \in W_{w}^{u n}, m, n \geq 0$ and (not necessarily pairwise distinct) $\bar{v}_{m} \in W^{m}$ and $\bar{u}_{n} \in W^{n}$ such that $v_{m} \prec \ldots \prec v_{1} \prec u_{1} \prec \ldots \prec u_{n}$, and:

$$
\begin{aligned}
& \alpha=\gamma \frown \bar{v}_{m} \\
& \beta=\gamma \succ \bar{u}_{n} .
\end{aligned}
$$

But then, let $p \in \Theta$ be chosen arbitrarily. We reason as follows:

$$
\begin{aligned}
\alpha \in V_{w}^{u n}(p) & \Leftrightarrow v_{m} \in V(p) \\
& \Rightarrow u_{n} \in V(p) \quad \text { (by transitivity of } \prec \text { and monotonicity of } V \text { ) } \\
& \Leftrightarrow \beta \in V_{w}^{u n}(p)
\end{aligned}
$$

Lemma 7. Let $(\mathcal{M}, w)$ be a pointed $\Theta$-model. Then: 
(1) The relation $B$ defined as follows:

$$
B:=\left\{\left(w_{n}, \bar{w}_{n}\right),\left(\bar{w}_{n}, w_{n}\right) \mid \bar{w}_{n} \in W_{w}^{u n}\right\} .
$$

is a bi-asimulation from $\left(\mathcal{M}_{w}^{u n}, w\right)$ to $(\mathcal{M}, w)$ and from $(\mathcal{M}, w)$ to $\left(\mathcal{M}_{w}^{u n}, w\right)$;

(2) $T h_{B I L}\left(\mathcal{M}, v_{k}\right)=T h_{B I L}\left(\mathcal{M}, \bar{v}_{k}\right)$ for any $\bar{v}_{k} \in W_{w}^{u n}$;

(3) $T h_{B I L}\left(\mathcal{M}, \bar{u}_{n}\right)=T h_{B I L}\left(\mathcal{M}, \bar{v}_{k}\right)$ for any $\bar{u}_{n}, \bar{v}_{k} \in W_{w}^{u n}$ whenever $u_{n}=v_{k}$.

Proof. (Part 1) It is clear that $B$ satisfies conditions (elem), w-type, and (s-atom); the latter two conditions are even satisfied both from $\left(\mathcal{M}_{w}^{u n}, w\right)$ to $(\mathcal{M}, w)$ and from $(\mathcal{M}, w)$ to $\left(\mathcal{M}_{w}^{u n}, w\right)$. We consider the remaining conditions:

Condition (s-back). If $\bar{w}_{n} \in W_{w}^{u n}$ and $w_{n} \prec v$, then two cases are possible:

Case 1. $w_{n}=v$. Then we note that we have $\bar{w}_{n} \prec_{w}^{u n} \bar{w}_{n}, v B \bar{w}_{n}$ and $\bar{w}_{n} B v$.

Case 2. $w_{n} \neq v$. Then we must have $\left(\bar{w}_{n}\right)^{-} v \in W_{w}^{u n}, \bar{w}_{n} \prec_{w}^{u n}\left(\bar{w}_{n}\right)^{-} v, v B\left(\bar{w}_{n}\right)^{-} v$, and $\left(\bar{w}_{n}\right) \frown v B v$.

On the other hand, if $\bar{w}_{n} \prec_{w}^{u n} \alpha$ for some $\alpha \in W_{w}^{u n}$ then it follows, by Lemma 5 , that we can choose some $m_{0}, n_{0} \geq 0$ and $\gamma \in W_{w}^{u n}$ and (not necessarily pairwise distinct) $\bar{v}_{m_{0}} \in W^{m_{0}}$ and $\bar{u}_{n_{0}} \in W^{n_{0}}$ such that $v_{m_{0}} \prec \ldots \prec v_{1} \prec e n d(\gamma) \prec u_{1} \prec \ldots \prec u_{n_{0}}$, and:

$$
\begin{aligned}
\bar{w}_{n} & =\gamma\left\ulcorner\bar{v}_{m_{0}}\right. \\
\alpha & =\gamma\left\ulcorner\bar{u}_{n_{0}} .\right.
\end{aligned}
$$

Again, two cases are possible:

Case 1. $m_{0}+n_{0}=0$. Then $\bar{w}_{n}=\alpha$; note, further, that we have then $w_{n} \prec w_{n}$, $w_{n} B \alpha$ and $\alpha B w_{n}$.

Case 2. $m_{0}+n_{0}>0$. Then (depending on whether $m_{0}=0$ or $m_{0} \neq 0$ ) we must have that $w_{n}=\operatorname{end}(\gamma)$ or $w_{n}=v_{m_{0}}$. In both cases it follows, by transitivity of $\prec$, that we have $w_{n} \prec u_{n_{0}}$; on the other hand, we have both $u_{n_{0}} B \alpha$ and $\alpha B u_{n_{0}}$.

Condition (s-forth). If $\bar{w}_{n} \in W_{w}^{u n}$ and $w_{n} \succ v$, then two cases are possible:

Case 1. $w_{n}=v$. Then we note that we have $\bar{w}_{n} \prec_{w}^{u n} \bar{w}_{n}, v B \bar{w}_{n}$ and $\bar{w}_{n} B v$.

Case 2. $w_{n} \neq v$. Then we must have $\left(\bar{w}_{n}\right) \frown v \in W_{w}^{u n},\left(\bar{w}_{n}\right) \frown v \prec_{w}^{u n} \bar{w}_{n}, v B\left(\bar{w}_{n}\right) \frown v$, and $\left(\bar{w}_{n}\right) \frown v B v$.

On the other hand, if $\alpha \prec_{w}^{u n} \bar{w}_{n}$ for some $\alpha \in W_{w}^{u n}$ then it follows, by Lemma 5 , that we can choose some $m_{0}, n_{0} \geq 0$ and $\gamma \in W_{w}^{u n}$ such that and (not necessarily pairwise distinct) $\bar{v}_{m_{0}} \in W^{m_{0}}$ and $\bar{u}_{n_{0}} \in W^{n_{0}}$ such that $v_{m_{0}} \prec \ldots \prec v_{1} \prec e n d(\gamma) \prec u_{1} \prec \ldots \prec u_{n_{0}}$, and:

$$
\begin{gathered}
\alpha=\gamma \frown \bar{v}_{m_{0}} \\
\bar{w}_{n}=\gamma \frown \bar{u}_{n_{0}} .
\end{gathered}
$$

Again, two cases are possible:

Case 1. $m_{0}+n_{0}=0$. Then $\bar{w}_{n}=\alpha$; note, further, that we have then $w_{n} \prec w_{n}$, $w_{n} B \alpha$ and $\alpha B w_{n}$.

Case 2. $m_{0}+n_{0}>0$. Then (depending on whether $n_{0}=0$ or $n_{0} \neq 0$ ) we must have that $w_{n}=\operatorname{end}(\gamma)$ or $w_{n}=u_{n_{0}}$. In both cases it follows, by transitivity of $\prec$, that we have $w_{n} \succ v_{m_{0}}$; on the other hand, we have both $v_{m_{0}} B \alpha$ and $\alpha B v_{m_{0}}$.

Parts 2 and 3 then follow from preservation of bi-intuitionistic formulas under biasimulations.

More generally, we will call an arbitrary $(\mathcal{M}, w) \in \operatorname{Pmod}_{\Theta}$ a bi-unravelled model iff there is an $(\mathcal{N}, v) \in \operatorname{Pmod}_{\Theta}$ and a bijection $g: W \rightarrow U^{u n(v)}$ satisfying the condition (i-rel) of Definition 1 such that $g(v)=w$; or, in other words, if the underlying frame 
$(W, \prec)$ of $(\mathcal{M}, w)$ is isomorphic to the underlying frame of $\left(\mathcal{N}^{u n(v)}, v\right)$. It is clear that if $\mathcal{M}$ is a bi-unravelled model, we may always assume that its underlying frame is just a copy of the underlying frame of the respective bi-unravelled model. We will assume, therefore, for the sake of simplicity, that if $(\mathcal{M}, w) \in \operatorname{Prod}_{\Theta}$ is a bi-unravelled model, then $\mathcal{M}$ is given in the form $\left\langle W^{u n(w)}, \prec^{u n(w)}, V\right\rangle$.

\section{Abstract Bi-Intuitionistic LOGiCs}

An abstract bi-intuitionistic logic $\mathcal{L}$ is a pair $\left(L, \models_{\mathcal{L}}\right)$, where $L$ maps every signature $\Theta$ to the set $L(\Theta)$ of $\Theta$-formulas of $\mathcal{L}$ and $\models_{\mathcal{L}}$ is a class-relation such that, if $\alpha \models_{\mathcal{L}} \beta$, then there exists a signature $\Theta$ such that $\alpha \in \operatorname{Pmod}_{\Theta}$, and $\beta \in L(\Theta)$; informally this is to mean that $\beta$ holds in $\alpha$. The relation $\models_{\mathcal{L}}$ is only assumed to be defined (i.e. to either hold or fail) for the elements of the class $\bigcup\left\{\operatorname{Pmod}_{\Theta} \times L(\Theta) \mid \Theta\right.$ is a signature $\}$ and to be undefined otherwise.

In order for such a pair $\mathcal{L}$ to count as an abstract bi-intuitionistic logic, we demand that the following conditions are satisfied:

- $\Theta \subseteq \Theta^{\prime} \Rightarrow L(\Theta) \subseteq L\left(\Theta^{\prime}\right)$.

- (Isomorphism). If $(\mathcal{M}, w) \in \operatorname{Pmod}_{\Theta}$ and $\mathcal{N} \in \operatorname{Mod}_{\Theta}, \phi \in L(\Theta)$ and $f: \mathcal{M} \cong \mathcal{N}$, then:

$$
\mathcal{M}, w \models_{\mathcal{L}} \phi \Leftrightarrow \mathcal{N}, f(w) \models_{\mathcal{L}} \phi .
$$

- (Expansion). If $\Theta$ is a signature, $\phi \in L(\Theta), \Theta \subseteq \Theta^{\prime}$, and $\mathcal{M}$ is a $\Theta^{\prime}$-model, then:

$$
\mathcal{M}, w \models_{\mathcal{L}} \phi \Leftrightarrow \mathcal{M} \uparrow \Theta, w \models_{\mathcal{L}} \phi .
$$

- (Occurrence). If $\phi \in L(\Theta)$ for some signature, then there is a finite $\Theta_{\phi} \subseteq \Theta$ such that for every $\Theta^{\prime}$-model $\mathcal{M}$, the relation $\mathcal{M} \models_{\mathcal{L}} \phi$ is defined iff $\Theta_{\phi} \subseteq \Theta^{\prime}$.

- (Closure). For every signature $\Theta$ and all $\phi, \psi \in L(\Theta)$, we have

$$
\perp, \phi \ll \psi, \phi \rightarrow \psi, \phi \wedge \psi, \phi \vee \psi \in L(\Theta),
$$

that is to say, $\mathcal{L}$ always includes falsum and is closed under intuitionistic implication, co-implication, conjunction and disjunction.

We further define that given a pair of abstract intuitionistic logics $\mathcal{L}$ and $\mathcal{L}^{\prime}$, we say that $\mathcal{L}^{\prime}$ extends $\mathcal{L}$ and write $\mathcal{L} \sqsubseteq \mathcal{L}^{\prime}$ when for all signatures $\Theta$ and $\phi \in L(\Theta)$ there exists a $\psi \in L^{\prime}(\Theta)$ such that for arbitrary pointed $\Theta$-model $(\mathcal{M}, w)$ it is true that:

$$
\mathcal{M}, w \models_{\mathcal{L}} \phi \Leftrightarrow \mathcal{M}, w \models \mathcal{L}^{\prime} \psi \text {. }
$$

If both $\mathcal{L} \sqsubseteq \mathcal{L}^{\prime}$ and $\mathcal{L}^{\prime} \sqsubseteq \mathcal{L}$ holds, then we say that the logics $\mathcal{L}$ and $\mathcal{L}^{\prime}$ are expressively equivalent and write $\mathcal{L} \equiv \mathcal{L}^{\prime}$.

It is easy to see that bi-intuitionistic propositional logic itself turns out to be an abstract bi-intuitionistic logic BIL $=\left(B I L, \models_{B I L}\right)$ under this definition. It is also obvious that the above definitions and conventions about bi-intuitionistic theories can be carried over to an arbitrary abstract bi-intuitionistic logic $\mathcal{L}$ replacing everywhere $B I L$ with $\mathcal{L}$, including such notions as $\mathcal{L}$-elementary submodel, $\mathcal{L}$-elementary embedding, $\mathcal{L}$-type, $\mathcal{L}$-saturation of a model, etc.

In this paper, our specific interest is in the extensions of BIL. Since every abstract intuitionistic logic $\mathcal{L}$ extending BIL must have an equivalent for every bi-intuitionistic 
propositional formula, we will just assume that for every signature $\Theta$ we have $B I L(\Theta) \subseteq L(\Theta)$ and that for every $\varphi \in B I L(\Theta)$ and every pointed $\Theta$-model $(\mathcal{M}, w)$ we have that:

$$
\mathcal{M}, w \models_{\mathcal{L}} \varphi \Leftrightarrow \mathcal{M}, w \models_{B I L} \varphi,
$$

so that all the bi-intuitionistic propositional formulas are present in $\mathcal{L}$ in their usual form and with their usual meaning, and whatever other formulas that $\mathcal{L}$ may contain are distinct from the elements of $B I L(\Theta)$.

We can immediately state the following corollary to Lemma3 for arbitrary extensions of BIL:

Corollary 2. Let $\mathrm{BIL} \sqsubseteq \mathcal{L}$, and let $\left(\mathcal{M}_{1}, w_{1}\right),\left(\mathcal{M}_{2}, w_{2}\right)$ be two pointed $\Theta$-models. If $T h_{B I L}^{+}\left(\mathcal{M}_{1}, w_{1}\right) \subseteq T h_{B I L}^{+}\left(\mathcal{M}_{2}, w_{2}\right)$ and both $\mathcal{M}_{1}$ and $\mathcal{M}_{2}$ are $\mathcal{L}$-saturated, then the relation $A$ such that for all $u \in W_{i}, s \in W_{j}$ if $\{i, j\}=\{1,2\}$, then

$$
u A s \Leftrightarrow\left(T h_{B I L}^{+}\left(\mathcal{M}_{i}, u\right) \subseteq T h_{B I L}^{+}\left(\mathcal{M}_{j}, s\right)\right)
$$

is a bi-asimulation from $\left(\mathcal{M}_{1}, w_{1}\right)$ to $\left(\mathcal{M}_{2}, w_{2}\right)$.

To prove this, we just repeat the proof of Lemma 3 using the fact that every $\mathcal{L}$ saturated model is of course $B I L$-saturated.

Some of the extensions of BIL turn out to be better than others in that they have useful model-theoretic properties. We define some of the relevant properties below.

Definition 7. Let $\mathcal{L}=\left(L, \models_{\mathcal{L}}\right)$ be an abstract bi-intuitionistic logic. Then:

- $\mathcal{L}$ is preserved under bi-asimulations, iff for every signature $\Theta$ and for arbitrary pointed $\Theta$-models $\left(\mathcal{M}_{1}, w_{1}\right)$ and $\left(\mathcal{M}_{2}, w_{2}\right)$, whenever $A$ is a bi-asimulation from $\left(\mathcal{M}_{1}, w_{1}\right)$ to $\left(\mathcal{M}_{2}, w_{2}\right)$, then the inclusion $T h_{\mathcal{L}}^{+}\left(\mathcal{M}_{1}, w_{1}\right) \subseteq T h_{\mathcal{L}}^{+}\left(\mathcal{M}_{2}, w_{2}\right)$ holds.

- $\mathcal{L}$ is $\star$-compact, iff an arbitrary $L(\Theta)$-theory $(\Gamma, \Delta)$ is $\mathcal{L}$-satisfiable, whenever for every finite $\Gamma^{\prime} \subseteq \Gamma$ and $\Delta^{\prime} \subseteq \Delta$, the theory $\left(\Gamma^{\prime}, \Delta^{\prime}\right)$ is $\mathcal{L}$-satisfiable.

- $\mathcal{L}$ has Tarski Union Property (TUP) iff for every $\mathcal{L}$-elementary chain

$$
\mathcal{M}_{0} \preccurlyeq \mathcal{L}, \ldots, \preccurlyeq \mathcal{L} \mathcal{M}_{n} \preccurlyeq \mathcal{L}, \ldots
$$

it is true that:

$$
\mathcal{M}_{n} \preccurlyeq \mathcal{L} \bigcup_{n \in \omega} \mathcal{M}_{n}
$$

for all $n \in \omega$.

If an abstract bi-intuitionistic logic is preserved under bi-asimulations, then every formula of this logic is monotonic w.r.t. accessibility relation. More precisely, the following lemma holds:

Lemma 8. If $\mathcal{L}$ is an abstract bi-intuitionistic logic that is preserved under bi-asimulations, then for every pointed $\Theta$-model $(\mathcal{M}, w)$, for every $v \in W$ such that $w \prec v$, and for every $\phi \in L(\Theta)$ it is true that:

$$
\mathcal{M}, w \models_{\mathcal{L}} \phi \Rightarrow \mathcal{M}, v \models_{\mathcal{L}} \phi .
$$

Proof. We define a bi-asimulation $A$ from $(\mathcal{M}, w)$ to $(\mathcal{M}, v)$ setting:

$$
A:=\{(w, v)\} \cup i d_{W} .
$$

We show that $A$ is indeed a bi-asimulation. Clearly, we only need to check conditions (s-back) and (s-forth). Moreover, if $s A t$ are such that $s=t$, then even the latter two 
conditions are clearly satisfied. There remains the case when $s=w$ and $t=v$. In this case, if $v \prec u$, then also $w \prec u$ by transitivity and we can also choose $u$ as a successor for $w$. Symmetrically, if $u \prec w$, then, by transitivity, also $u \prec v$; thus we can also choose $u$ as a predecessor for $v$.

The lemma then follows by the preservation of $\mathcal{L}$ under bi-asimulations.

Note that for Lemma 8 one does not even need to assume that BIL $\sqsubseteq \mathcal{L}$. The next lemma sums up some well-known facts about BIL:

Lemma 9. BIL is preserved under bi-asimulations, $\star$-compact and has TUP.

Proof. Invariance under bi-asimulations follows from the main result of [1]. The $\star-$ compactness of BIL is follows from the fact that it is representable as a fragment of classical first-order logic by the appropriate standard translation (also in [1). The proof of TUP runs along the lines of standard proofs of TUP; we briefly sketch it here.

We need to show that if $\varphi \in B I L(\Theta), n \in \omega$ and $w \in W_{n}$, then

$$
\mathcal{M}_{n}, w \models_{B I L} \varphi \Leftrightarrow \bigcup_{n \in \omega} \mathcal{M}_{n}, w \models_{B I L} \varphi ;
$$

this is done by induction on the construction of $\varphi$, and the only non-trivial cases are is when $\varphi=\psi \rightarrow \chi$ and when $\varphi=\psi \ll \chi$.

Case 1. Assume that $\varphi=\psi \rightarrow \chi$. If $\mathcal{M}_{n}, w \nvdash_{B I L} \psi \rightarrow \chi$, then there is a $v \in W_{n}$ such that $w \prec_{n} v$ and $\mathcal{M}_{n}, v \models_{B I L}(\{\psi\},\{\chi\})$. But then, by induction hypothesis we must have $\bigcup_{n \in \omega} \mathcal{M}_{n}, v \models_{B I L}(\{\psi\},\{\chi\})$ and we also have $w\left(\bigcup_{n \in \omega} \prec_{n}\right) v$ so that $\bigcup_{n \in \omega} \mathcal{M}_{n}, w \forall_{B I L} \psi \rightarrow \chi$. In the other direction, assume that $\bigcup_{n \in \omega} \mathcal{M}_{n}, w \nvdash_{B I L} \psi \rightarrow \chi$. Then, for some $v \in \bigcup_{n \in \omega} W_{n}$ such that $w\left(\bigcup_{n \in \omega} \prec n\right) v$ it is true that $\bigcup_{n \in \omega} \mathcal{M}_{n}, v \models_{B I L}(\{\psi\},\{\chi\})$. But then, for some $k \geq n$, we must have both $w, v \in W_{k}$ and $w \prec_{k} v$, so that we get $\mathcal{M}_{k}, w \forall_{B I L} \psi \rightarrow \chi$. By obvious transitivity of $\preccurlyeq B I L$ we get then that $\mathcal{M}_{n} \preccurlyeq B I L \mathcal{M}_{k}$, whence $\mathcal{M}_{n}, w \not \forall_{B I L} \psi \rightarrow \chi$.

Case 2. Assume that $\varphi=\psi \ll \chi$. If $\mathcal{M}_{n}, w \models_{B I L} \psi \ll \chi$, then there is a $v \in W_{n}$ such that $v \prec_{n} w$ and $\mathcal{M}_{n}, v \models_{B I L}(\{\psi\},\{\chi\})$. But then, by induction hypothesis we must have $\bigcup_{n \in \omega} \mathcal{M}_{n}, v \models_{B I L}(\{\psi\},\{\chi\})$ and we also have $v\left(\bigcup_{n \in \omega} \prec_{n}\right) w$ so that $\bigcup_{n \in \omega} \mathcal{M}_{n}, w \models_{B I L} \psi \ll \chi$. In the other direction, assume that $\bigcup_{n \in \omega} \mathcal{M}_{n}, w \models_{B I L} \psi \ll \chi$. Then, for some $v \in \bigcup_{n \in \omega} W_{n}$ such that $v\left(\bigcup_{n \in \omega} \prec_{n}\right) w$ it is true that $\bigcup_{n \in \omega} \mathcal{M}_{n}, v \models_{B I L}(\{\psi\},\{\chi\})$. But then, for some $k \geq n$, we must have both $w, v \in W_{k}$ and $v \prec_{k} w$, so that we get $\mathcal{M}_{k}, w \models_{B I L} \psi \ll \chi$. By obvious transitivity of $\preccurlyeq_{B I L}$ we get then that $\mathcal{M}_{n} \preccurlyeq B I L \mathcal{M}_{k}$, whence $\mathcal{M}_{n}, w \models_{B I L} \psi \ll \chi$.

The abstract bi-intuitionistic logics extending BIL and displaying the combination of all three useful model-theoretic properties mentioned in Lemma 9 indeed, display a very regular behavior. In particular, one easily checks that an obvious analogue of every Lemma and Corollary established in Sections 23 above can be established for any such logic without changing anything essential in the proofs.

Our main theorem is then that no proper extension of BIL displays the combination of useful properties established in Lemma 9. In other words, we are going to establish the following:

Theorem 1. Let $\mathcal{L}$ be an abstract bi-intuitionistic logic. If $\mathrm{BIL} \sqsubseteq \mathcal{L}$ and $\mathcal{L}$ is preserved under bi-asimulations, $\star$-compact, and has the TUP, then $\mathrm{BIL} \equiv \mathcal{L}$. 


\section{The Proof of Theorem 1}

Before we start with the proof, we need one more piece of notation. If $\mathcal{L}$ is an abstract bi-intuitionistic logic, $\Theta$ a signature, and $\Gamma \subseteq L(\Theta)$, then we let $\operatorname{Mod}_{\mathcal{L}}(\Theta, \Gamma)$ denote the class of pointed $\Theta$-models $(\mathcal{N}, u)$ such that for every $\phi \in \Gamma$ it is true that:

$$
\mathcal{N}, u \models_{\mathcal{L}} \phi .
$$

If $\Gamma=\{\phi\}$ for some $\phi \in L(\Theta)$ then instead of $\operatorname{Mod}_{\mathcal{L}}(\Theta, \Gamma)$ we simply write $\operatorname{Mod}_{\mathcal{L}}(\Theta, \phi)$.

We now start by establishing a couple of technical facts first:

Proposition 1. Let $\mathcal{L}$ be a $\star$-compact abstract bi-intuitionistic logic extending BIL. Suppose that $\mathrm{BIL} \not \equiv \mathcal{L}$. Then, there are $\phi \in L\left(\Theta_{\phi}\right)$ and pointed $\Theta_{\phi}$-models $\left(\mathcal{M}_{1}, w_{1}\right)$, $\left(\mathcal{M}_{2}, w_{2}\right)$ such that $T h_{B I L}^{+}\left(\mathcal{M}_{1}, w_{1}\right) \subseteq T h_{B I L}^{+}\left(\mathcal{M}_{2}, w_{2}\right)$ while $\mathcal{M}_{1}, w_{1} \models_{\mathcal{L}} \phi$ and $\mathcal{M}_{2}, w_{2} \nvdash_{\mathcal{L}} \phi$.

Proof. Suppose that for an arbitrary $\phi \in L\left(\Theta_{\phi}\right)$ we have shown that:

(i) $\operatorname{Mod}_{\mathcal{L}}\left(\Theta_{\phi}, \phi\right)=\bigcup_{(\mathcal{N}, u) \in \operatorname{Mod}_{\mathcal{L}}\left(\Theta_{\phi}, \phi\right)} \operatorname{Mod}_{\mathcal{L}}\left(\Theta_{\phi}, T h_{B I L}^{+}(\mathcal{N}, u)\right)$,

Let $(\mathcal{N}, u) \in \operatorname{Mod}_{\mathcal{L}}\left(\Theta_{\phi}, \phi\right)$ be arbitrary. The above implies that every $\Theta_{\phi}$-model of $T h_{B I L}^{+}(\mathcal{N}, u)$ must be a model of $\phi$. But then the theory $\left(T h_{B I L}^{+}(\mathcal{N}, u),\{\phi\}\right)$ is $\mathcal{L}$ unsatisfiable. By the $\star$-compactness of $\mathcal{L}$, for some finite $\Psi_{(\mathcal{N}, u)} \subseteq T h_{B I L}^{+}(\mathcal{N}, u)$ (and we can pick a unique one using the Axiom of Choice if need be), the theory $\left(\Psi_{(\mathcal{N}, u)},\{\phi\}\right)$ is $\mathcal{L}$-unsatisfiable. Hence, $\bigwedge \Psi_{(\mathcal{N}, u)}$ logically implies $\phi$ in $\mathcal{L}$. Then, given (i), we get that

(ii) $\operatorname{Mod}_{\mathcal{L}}\left(\Theta_{\phi}, \phi\right)=\bigcup_{(\mathcal{N}, u) \in \operatorname{Mod}_{\mathcal{L}}\left(\Theta_{\phi}, \phi\right)} \operatorname{Mod}_{\mathcal{L}}\left(\Theta_{\phi}, \Psi_{(\mathcal{N}, u)}\right)$.

However, this means that the theory $\left(\{\phi\},\left\{\bigwedge \Psi_{(\mathcal{N}, u)} \mid(\mathcal{N}, u) \in \operatorname{Mod}_{\mathcal{L}}\left(\Theta_{\phi}, \phi\right)\right\}\right)$ is $\mathcal{L}$ unsatisfiable and by (i), for some finite $\Gamma \subseteq\left\{\bigwedge \Psi_{(\mathcal{N}, u)} \mid(\mathcal{N}, u) \in \operatorname{Mod}_{\mathcal{L}}\left(\Theta_{\phi}, \phi\right)\right\}$, the theory $(\{\phi\}, \Gamma)$ is $\mathcal{L}$-unsatisfiable. This means that $\operatorname{Mod}_{\mathcal{L}}\left(\Theta_{\phi}, \phi\right) \subseteq \operatorname{Mod}_{\mathcal{L}}\left(\Theta_{\phi}, \bigvee \Gamma\right)$ So, using (ii), since clearly $\operatorname{Mod}_{\mathcal{L}}\left(\Theta_{\phi}, \bigvee \Gamma\right) \subseteq \bigcup_{(\mathcal{N}, u) \in \operatorname{Mod}_{\mathcal{L}}\left(\Theta_{\phi}, \phi\right)} \operatorname{Mod}_{\mathcal{L}}\left(\Theta_{\phi}, \Psi_{(\mathcal{N}, u)}\right)$, we get that:

(iii) $\operatorname{Mod}_{\mathcal{L}}\left(\Theta_{\phi}, \phi\right)=\operatorname{Mod}_{\mathcal{L}}\left(\Theta_{\phi}, \bigvee \Gamma\right)$

Now, $\bigvee \Gamma$ is a perfectly good formula of $B I L\left(\Theta_{\phi}\right)$ involving only finitary conjunctions and disjunctions. So we have shown that every $\phi \in L\left(\Theta_{\phi}\right)$ is just a bi-intuitionistic $\Theta_{\phi}$-formula and hence that $\mathcal{L} \equiv \mathrm{BIL}$ which is in contradiction with the hypothesis of the proposition.

Therefore, (i) must fail for at least one $\phi \in L\left(\Theta_{\phi}\right)$, and clearly, for this $\phi$ it can only fail if

$$
\bigcup_{(\mathcal{N}, u) \in \operatorname{Mod}_{\mathcal{L}}\left(\Theta_{\phi}, \phi\right)} \operatorname{Mod}_{\mathcal{L}}\left(\Theta_{\phi}, T h_{B I L}^{+}(\mathcal{N}, u)\right) \not \subset \operatorname{Mod}_{\mathcal{L}}\left(\Theta_{\phi}, \phi\right) .
$$

But the latter means that for some pointed intuitionistic $\Theta_{\phi}$-model $\left(\mathcal{M}_{1}, w_{1}\right)$ such that $\mathcal{M}_{1}, w_{1} \models_{\mathcal{L}} \phi$ there is another $\Theta_{\phi}$-model $\left(\mathcal{M}_{2}, w_{2}\right)$ such that both $\mathcal{M}_{2}, w_{2} \nvdash_{\mathcal{L}} \phi$ and $T h_{B I L}^{+}\left(\mathcal{M}_{1}, w_{1}\right)$ is satisfied at $\left(\mathcal{M}_{2}, w_{2}\right)$. The latter means, in turn, that we have $T h_{B I L}^{+}\left(\mathcal{M}_{1}, w_{1}\right) \subseteq T h_{B I L}^{+}\left(\mathcal{M}_{2}, w_{2}\right)$ as desired.

Assume $\mathcal{M}$ is a $\Theta$-model. We let $\Theta_{\mathcal{M}}$ be $\Theta \cup\left\{q_{w}^{+}, q_{w}^{-} \mid w \in W\right\}$ such that $\Theta \cap\left\{q_{w}^{+}, q_{w}^{-} \mid w \in W\right\}=\varnothing$, and we define that $[\mathcal{M}]=(W, \prec,[V])$ is the $\Theta_{\mathcal{M}}$-model, such that $W$ and $\prec$ are just borrowed from $\mathcal{M}$ and $[V]$ coincides with $V$ on elements 
of $\Theta$, whereas for arbitrary $v, w \in W$ we set that $v \in[V]\left(q_{w}^{+}\right)$iff $w \prec v$ and $v \notin[V]\left(q_{w}^{-}\right)$ iff $v \prec w$. It is straightforward to check that $[\mathcal{M}]$ is a $\Theta_{\mathcal{M}}$-model.

Lemma 10. Let $\mathcal{L}$ be an abstract bi-intuitionistic logic preserved under bi-asimulations and extending $\mathrm{BIL}$, let $(\mathcal{M}, w)$ be a pointed bi-unravelled $\Theta$-model, and let $(\mathcal{N}, v)$ be another pointed $\Theta_{\mathcal{M}}$-model. Assume that $T h_{\mathcal{L}}(\mathcal{N}, v)=T h_{\mathcal{L}}([\mathcal{M}], w)$. Then there exists an $\mathcal{L}$-elementary embedding $f$ of $[\mathcal{M}]$ into $\mathcal{N}$ such that $f(w)=v$.

Proof. We proceed by induction on $k \geq 1$ and define an increasing chain of the form

$$
f_{1} \subseteq \ldots \subseteq f_{k} \subseteq \ldots
$$

in such a way that, for each $k<\omega, f_{k}:\left\{\bar{u}_{l} \in W_{w}^{u n} \mid l \leq k\right\} \rightarrow U$ is an injective function. In the process of inductively defining $f_{k}$ for all $k<\omega$, we show that our construction satisfies the following conditions for all $1 \leq l, m \leq k<\omega$, and all $\bar{u}_{l}, \bar{v}_{m}, \bar{w}_{k} \in W_{w}^{u n}$ :

$(i)_{k} T h_{\mathcal{L}}\left(\mathcal{N}, f_{k}\left(\bar{w}_{k}\right)\right)=T h_{\mathcal{L}}\left([\mathcal{M}], \bar{w}_{k}\right)$.

$(i i)_{k} f_{k}\left(\bar{u}_{l}\right) \triangleleft f_{k}\left(\bar{v}_{m}\right) \Leftrightarrow \bar{u}_{l} \prec_{w}^{u n} \bar{v}_{m}$.

Induction basis. $k=1$. Then $\bar{w}_{k}=w$, so we have to define $f_{1}$ for this unique node. We set $f_{1}(w):=v$ thus getting an injective function. Under these settings, it is true that

$$
T h_{\mathcal{L}}\left(\mathcal{N}, f_{1}(w)\right)=T h_{\mathcal{L}}(\mathcal{N}, v)=T h_{\mathcal{L}}([\mathcal{M}], w) \quad(\text { by the assumption of the lemma })
$$

so $(i)_{1}$ is satisfied. Next, if $1 \leq l, m \leq 1$ and $\bar{u}_{l}, \bar{w}_{m} \in W_{w}^{u n}$, then $l=m=1$ and we get $\bar{u}_{l}=w=\bar{w}_{m}=\bar{w}_{l}$ so that both $\bar{u}_{l} \prec_{w}^{u n} \bar{w}_{m}$ and $f_{1}\left(\bar{u}_{l}\right)=v \triangleleft v=f_{1}\left(\bar{w}_{m}\right)$ by reflexivity. Therefore, $(i i)_{1}$ is satisfied as well.

Induction step. $k=l+1$ for some $l \geq 1$. Then we already have the chain of injections $f_{1} \subseteq \ldots \subseteq f_{l}$ defined in such a way that $(i)_{m}-(i i)_{m}$ are satisfied by $f_{m}$ for all $m \leq l$.

If $\bar{w}_{k} \in W_{w}^{u n}$ is chosen arbitrarily, then we must have $\bar{w}_{k}=\bar{w}_{l}^{-} w_{k}$. We have then

$$
T h_{\mathcal{L}}\left(\mathcal{N}, f_{l}\left(\bar{w}_{l}\right)\right)=T h_{\mathcal{L}}\left([\mathcal{M}], \bar{w}_{l}\right)
$$

by $(i)_{l}$. Two cases are possible:

Case 1. $w_{l} \prec w_{k}$. Then, by definition of $[\mathcal{M}]$ and by the closure of $\mathcal{L}$ w.r.t. intuitionistic implication, we have that the set:

$$
\Gamma_{\bar{w}_{k}}=\left\{q_{\bar{w}_{k}}^{+} \rightarrow \phi \mid \phi \in T h_{\mathcal{L}}^{+}\left([\mathcal{M}], \bar{w}_{k}\right)\right\} \cup\left\{\psi \rightarrow q_{\bar{w}_{k}}^{-} \mid \psi \in T h_{\mathcal{L}}^{-}\left([\mathcal{M}], \bar{w}_{k}\right)\right\}
$$

is a subset of $T h_{\mathcal{L}}^{+}\left([\mathcal{M}], \bar{w}_{l}\right)$, thus also of $T h_{\mathcal{L}}^{+}\left(\mathcal{N}, f_{l}\left(\bar{w}_{l}\right)\right)$. We also know that, by $\bar{w}_{l} \prec_{w}^{u n} \bar{w}_{k}$, and by the fact that the theory $\left(\left\{q_{\bar{w}_{k}}^{+}\right\},\left\{q_{\bar{w}_{k}}^{-}\right\}\right)$is $\mathcal{L}$-satisfied at $\left([\mathcal{M}], \bar{w}_{k}\right)$, we have:

$$
[\mathcal{M}], \bar{w}_{l} \not \nvdash_{\mathcal{L}} q_{\bar{w}_{k}}^{+} \rightarrow q_{\bar{w}_{k}}^{-} .
$$

Therefore, by (11), also $\mathcal{N}, f_{l}\left(\bar{w}_{l}\right) \not \nvdash_{\mathcal{L}} q_{\bar{w}_{k}}^{+} \rightarrow q_{\bar{w}_{k}}^{\bar{w}_{k}}$. So fix any $v_{\bar{w}_{k}}^{\prime} \in U$ such that $f_{l}\left(\bar{w}_{l}\right) \triangleleft v_{\bar{w}_{k}}^{\prime}$ and $\left(\left\{q_{\bar{w}_{k}}^{+}\right\},\left\{q_{\bar{w}_{k}}^{-}\right\}\right)$is $\mathcal{L}$-satisfied at $\left(\mathcal{N}, v_{\bar{w}_{k}}^{\prime}\right)$. By Lemma 8 and the preservation of $\mathcal{L}$ under bi-asimulations, we get that $\Gamma_{\bar{w}_{k}} \subseteq T h_{\mathcal{L}}^{+}\left(\mathcal{N}, f_{l}\left(\bar{w}_{l}\right)\right) \subseteq T h_{\mathcal{L}}^{+}\left(\mathcal{N}, v_{\bar{w}_{k}}^{\prime}\right)$ and thus, clearly, $T h_{\mathcal{L}}\left([\mathcal{M}], \bar{w}_{k}\right)=T h_{\mathcal{L}}\left(\mathcal{N}, v_{\bar{w}_{k}}^{\prime}\right)$.

Case 2. $w_{l} \succ w_{k}$. Then, by definition of $[\mathcal{M}]$ and by the closure of $\mathcal{L}$ w.r.t. coimplication, we have that the set:

$$
\Delta_{\bar{w}_{k}}=\left\{q_{\bar{w}_{k}}^{+} \ll \phi \mid \phi \in T h_{\mathcal{L}}^{+}\left([\mathcal{M}], \bar{w}_{k}\right)\right\} \cup\left\{\psi \ll q_{\bar{w}_{k}}^{-} \mid \psi \in T h_{\mathcal{L}}^{-}\left([\mathcal{M}], \bar{w}_{k}\right)\right\}
$$


is a subset of $T h_{\mathcal{L}}^{-}\left([\mathcal{M}], \bar{w}_{l}\right)$, thus also of $T h_{\mathcal{L}}^{-}\left(\mathcal{N}, f_{l}\left(\bar{w}_{l}\right)\right)$. We also know that, by $\bar{w}_{k} \prec_{w}^{u n} \bar{w}_{l}$, and by the fact that the theory $\left(\left\{q_{\bar{w}_{k}}^{+}\right\},\left\{q_{\bar{w}_{k}}^{-}\right\}\right)$is $\mathcal{L}$-satisfied at $\left([\mathcal{M}], \bar{w}_{k}\right)$, we have:

$$
[\mathcal{M}], \bar{w}_{l} \models_{\mathcal{L}} q_{\bar{w}_{k}}^{+} \ll q_{\bar{w}_{k}}^{-} .
$$

Therefore, by (10), also $\mathcal{N}, f_{l}\left(\bar{w}_{l}\right) \models_{\mathcal{L}} q_{\bar{w}_{k}}^{+} \ll q_{\bar{w}_{k}}^{-}$. So fix any $v_{\bar{w}_{k}}^{\prime} \in U$ such that $f_{l}\left(\bar{w}_{l}\right) \triangleright v_{\bar{w}_{k}}^{\prime}$ and $\left(\left\{q_{\bar{w}_{k}}^{+}\right\},\left\{q_{\bar{w}_{k}}\right\}\right)$ is $\mathcal{L}$-satisfied at $\left(\mathcal{N}, v_{\bar{w}_{k}}^{\prime}\right)$. By Lemma 8 and the preservation of $\mathcal{L}$ under bi-asimulations, we get that $\Delta_{\bar{w}_{k}} \subseteq T h_{\mathcal{L}}^{-}\left(\mathcal{N}, f_{l}\left(\bar{w}_{l}\right)\right) \subseteq T h_{\mathcal{L}}^{-}\left(\mathcal{N}, v_{\bar{w}_{k}}^{\prime}\right)$ and thus, clearly, $T h_{\mathcal{L}}\left([\mathcal{M}], \bar{w}_{k}\right)=T h_{\mathcal{L}}\left(\mathcal{N}, v_{\bar{w}_{k}}^{\prime}\right)$.

Having thus decided on our choices for $v_{\bar{w}_{k}}^{\prime}$ for every $\bar{w}_{k} \in W_{w}^{u n}$, we now set:

$$
f_{k}\left(\bar{u}_{m}\right):= \begin{cases}f_{l}\left(\bar{u}_{m}\right), & \text { if } m \leq l \\ v_{\bar{u}_{m}}^{\prime}, & \text { if } m=k .\end{cases}
$$

It is clear then that $f_{k}$ is a function from $\left\{\bar{u}_{m} \in W_{w}^{u n} \mid m \leq k\right\}$ to $U$, since $f_{l}$ is a function and we have fixed a unique $v_{\bar{w}_{k}}^{\prime} \in U$ for every $\bar{w}_{k} \in W_{w}^{u n}$. It is also immediate to see that $(i)_{k}$ is satisfied.

We now address the injectivity of $f_{k}$. Let $m \leq r \leq k$ and let $\bar{u}_{m}, \bar{w}_{r} \in W_{w}^{u n}$ be such that $\bar{u}_{m} \neq \bar{w}_{r}$. Then we have either $\bar{u}_{m} \nprec_{w}^{u n} \bar{w}_{r}$ or $\bar{w}_{r} \nprec_{w}^{u n} \bar{u}_{m}$. In the first case, we have $q_{\bar{u}_{m}}^{+} \in T h_{\mathcal{L}}^{+}\left([\mathcal{M}], \bar{u}_{m}\right) \backslash T h_{\mathcal{L}}^{+}\left([\mathcal{M}], \bar{w}_{r}\right)$, so by $(i)_{k}$ we must have

$$
q_{\bar{u}_{m}}^{+} \in T h_{\mathcal{L}}^{+}\left(\mathcal{N}, f_{k}\left(\bar{u}_{m}\right)\right) \backslash T h_{\mathcal{L}}^{+}\left(\mathcal{N}, f_{k}\left(\bar{w}_{r}\right)\right),
$$

whence $f_{k}\left(\bar{u}_{m}\right) \neq f_{k}\left(\bar{w}_{k}\right)$. In the second case, we reason symmetrically, using $q_{\bar{w}_{r}}^{+}$ instead of $q_{\bar{u}_{m}}^{+}$. Thus $f_{k}$ is injective.

Next, we verify $(i i)_{k}$. Let $\bar{u}_{m}, \bar{w}_{r} \in W_{w}^{u n}$ be such that $m, r \leq k$.

$(\Leftarrow)$. If $\bar{u}_{m} \prec_{w}^{u n} \bar{w}_{r}$, then choose, by Lemma $5, m_{1}, n_{1} \geq 0$ and $\delta \in W_{w}^{u n}$ and (not necessarily pairwise distinct) $\bar{s}_{m_{1}} \in W^{m_{1}}$ and $\bar{t}_{n_{1}} \in W^{n_{1}}$ such that $s_{m_{1}} \prec \ldots \prec s_{1} \prec \operatorname{end}(\delta) \prec t_{1} \prec \ldots \prec t_{n_{1}}$, and:

$$
\begin{aligned}
\bar{u}_{m} & =\delta \smile \bar{s}_{m_{1}} \\
\bar{w}_{r} & =\delta \frown \bar{t}_{n_{1}} .
\end{aligned}
$$

The following cases are then possible:

Case 1. $m_{1}=n_{1}=0$. Then $\bar{u}_{m}=\bar{w}_{r}$, thus also $f\left(\bar{u}_{m}\right)=f\left(\bar{w}_{r}\right)$, and so $f\left(\bar{u}_{m}\right) \triangleleft f\left(\bar{w}_{r}\right)$ by reflexivity.

Case 2. $m_{1}, n_{1}>0$. But then also $\delta \frown \bar{s}_{m_{1}-1}, \delta \frown \bar{t}_{n_{1}-1} \in W_{w}^{u n}$, and due to their smaller length, we also get that $\delta^{\frown} \bar{s}_{m_{1}-1}, \delta^{\frown} \bar{t}_{n_{1}-1} \in \operatorname{dom}\left(f_{k}\right)$. Moreover, it follows from Lemma 5 that we have:

$$
\bar{u}_{m}=\delta \frown \bar{s}_{m_{1}} \prec_{w}^{u n} \delta \frown \bar{s}_{m_{1}-1} \prec_{w}^{u n} \delta \frown \bar{t}_{n_{1}-1} \prec \prec_{w}^{u n} \delta \frown \bar{t}_{n_{1}}=\bar{w}_{r} .
$$

We now claim the following:

Claim. We have:

$$
f_{k}\left(\bar{u}_{m}\right)=f_{k}\left(\delta \frown \bar{s}_{m_{1}}\right) \triangleleft f_{k}\left(\delta \frown \bar{s}_{m_{1}-1}\right) \triangleleft f_{k}\left(\delta \frown \bar{t}_{n_{1}-1}\right) \triangleleft f_{k}\left(\delta \frown \bar{t}_{n_{1}}\right)=f_{k}\left(\bar{w}_{r}\right) .
$$

Indeed, if $m=k$, then we have $f_{k}\left(\bar{u}_{m}\right)=f_{k}\left(\delta^{\frown} \bar{s}_{m_{1}}\right) \triangleleft f_{k}\left(\delta^{\frown} \bar{s}_{m_{1}-1}\right)$ by the choice of $f_{k}\left(\bar{u}_{m}\right)$, otherwise, we get it by $(i i)_{l}$. Similarly, if $r=k$, then we get $f_{k}\left(\delta \frown \bar{t}_{n_{1}-1}\right) \triangleleft f_{k}\left(\delta \frown \bar{t}_{n_{1}}\right)=f_{k}\left(\bar{w}_{r}\right)$ by the choice of $f_{k}\left(\bar{w}_{r}\right)$, otherwise we get the same result by $(i i)_{l}$. Finally, the length of both $\delta \frown \bar{s}_{m_{1}-1}$ and $\delta \frown \bar{t}_{n_{1}-1}$ cannot exceed $l$, therefore $f_{k}\left(\delta^{\frown} \bar{s}_{m_{1}-1}\right) \triangleleft f_{k}\left(\delta \frown \bar{t}_{n_{1}-1}\right)$ also follows from $(i i)_{l}$.

The Claim is thus proven and the $(\Leftarrow)$-part for Case 2 clearly follows from the Claim. 
Case 3. $m_{1}=0, n_{1}>0$. Then also $\bar{u}_{m}=\delta, \delta \frown \bar{t}_{n_{1}-1} \in W_{w}^{u n}$, and both of these sequences have a length that does not exceed $l$. We have then, by Lemma 5 that: $\bar{u}_{m}=\delta \prec_{w}^{u n} \delta \frown \bar{t}_{n_{1}-1} \prec_{w}^{u n} \delta \frown \bar{t}_{n_{1}}=\bar{w}_{r}$. We immediately get that $f_{k}\left(\bar{u}_{m}\right)=f_{l}\left(\bar{u}_{m}\right) \triangleleft f_{l}\left(\delta \frown \bar{t}_{n_{1}}\right)=f_{k}\left(\delta \frown \bar{t}_{n_{1}}\right)$ by $(i i)_{l}$. Now, if $r=k$, then we get $f_{k}\left(\delta \frown \bar{t}_{n_{1}-1}\right) \triangleleft f_{k}\left(\delta \frown \bar{t}_{n_{1}}\right)=f_{k}\left(\bar{w}_{r}\right)$ by the choice of $f_{k}\left(\bar{w}_{r}\right)$, otherwise we get the same result by $(i i)_{l}$. Summing up, we get that $f_{k}\left(\bar{u}_{m}\right) \triangleleft f_{k}\left(\delta \frown \bar{t}_{n_{1}}\right) \triangleleft f_{k}\left(\bar{w}_{r}\right)$, hence also $f_{k}\left(\bar{u}_{m}\right) \triangleleft f_{k}\left(\bar{w}_{r}\right)$ by transitivity.

Case 4. $m_{1}>0, n_{1}=0$. This case is symmetric to Case 4 .

$(\Rightarrow)$. If $f_{k}\left(\bar{u}_{m}\right) \triangleleft f_{k}\left(\bar{w}_{r}\right)$, then we have $q_{\bar{u}_{m}}^{+} \in T h_{\mathcal{L}}^{+}\left([\mathcal{M}], \bar{u}_{m}\right)=T h_{\mathcal{L}}^{+}\left(\mathcal{N}, f_{k}\left(\bar{u}_{m}\right)\right)$ by $(i)_{k}$, but then, by Lemma 8 , preservation of $\mathcal{L}$ under bi-asimulations, and $(i)_{k}$ again, we further get that

$$
q_{\bar{u}_{m}}^{+} \in T h_{\mathcal{L}}^{+}\left(\mathcal{N}, f_{k}\left(\bar{w}_{r}\right)\right)=T h_{\mathcal{L}}^{+}\left([\mathcal{M}], \bar{w}_{r}\right),
$$

whence we must have $\bar{u}_{m} \prec_{w}^{u n} \bar{w}_{r}$ by the definition of $[\mathcal{M}]$.

In this way, $(i i)_{k}$ is shown to hold and the Induction Step is proven.

We now define:

$$
f:=\bigcup\left\{f_{k} \mid k<\omega\right\}
$$

We will show that $f$ is an $\mathcal{L}$-elementary embedding of $[\mathcal{M}]$ into $\mathcal{N}$. It is obvious that $f: W_{w}^{u n} \rightarrow U$ is an injection, being a union of the countable increasing chain of injections such that the sequence of the domains of these injections covers all of $W_{w}^{u n}$.

It remains to check that the conditions of Definition 2 are satisfied by $f$. As for condition (rel), assume that $\bar{u}_{m}, \bar{w}_{k} \in W_{w}^{u n}$. Then we have

$$
\begin{aligned}
\bar{u}_{m} \prec_{w}^{u n} \bar{w}_{k} & \Leftrightarrow f_{\max (m, k)}\left(\bar{u}_{m}\right) \triangleleft f_{\max (m, k)}\left(\bar{w}_{k}\right) & & \left(\text { by }(i i)_{\max (m, k)}\right) \\
& \Leftrightarrow f\left(\bar{u}_{m}\right) \triangleleft f\left(\bar{w}_{k}\right) & & \text { (by definition of } f \text { ) }
\end{aligned}
$$

Finally, as for the condition (theories), we observe that for any given $\bar{u}_{m} \in W_{w}^{u n}$ and any $\phi \in L\left(\Theta_{\mathcal{M}}\right)$ we have:

$$
\begin{aligned}
T h_{\mathcal{L}}\left([\mathcal{M}], \bar{u}_{m}\right) & =T h_{\mathcal{L}}\left(\mathcal{N}, f_{m}\left(\bar{u}_{m}\right)\right) & & \left(\text { by }(i)_{m}\right) \\
& =T h_{\mathcal{L}}\left(\mathcal{N}, f\left(\bar{u}_{m}\right)\right) & & (\text { by definition of } f)
\end{aligned}
$$

More generally, extending the signature of a bi-unravelled $(\mathcal{M}, w) \in \operatorname{Pmod}_{\Theta}$ to get $[\mathcal{M}]$ allows us to force many types of formulas holding and failing at every node of $[\mathcal{M}]$ while staying at $w$. This functionality even extends to arbitrary models for which a suitable elementary embedding of $[\mathcal{M}]$ into them is possible. More precisely, the following lemma holds:

Lemma 11. Let $\mathcal{L}$ be an abstract bi-intuitionistic logic which extends BIL, is preserved under bi-asimulations, $\star$-compact, and has TUP, let $(\mathcal{M}, w) \in \operatorname{Pmod}_{\Theta}$ be a bi-unravelled model, and let $f$ be an $\mathcal{L}$-elementary embedding of $[\mathcal{M}]$ into $\mathcal{N}$. Then, for every $n>0$, every $\bar{w}_{n} \in W_{w}^{u n}$, and every $1 \leq k \leq n$ there exist binary formula schemas $\phi_{\bar{w}_{n}}^{k}\left(\__{1}, \__{2}\right), \psi_{\bar{w}_{n}}^{k}\left(\__{1}, \__{2}\right), \theta_{\bar{w}_{n}}^{k}\left(\__{1}, \__{2}\right), \tau_{\bar{w}_{n}}^{k}\left(\__{1}, \__{2}\right) \in B I L\left(\Theta_{\mathcal{M}}\right)$, and a function

$$
F:\left\{\phi_{\bar{w}_{n}}^{k}, \psi_{\bar{w}_{n}}^{k}, \theta_{\bar{w}_{n}}^{k}, \tau_{\bar{w}_{n}}^{k} \mid \bar{w}_{n} \in W_{w}^{u n}, 1 \leq k \leq n\right\} \rightarrow\{+,-\}
$$

such that, if $1 \leq k \leq n$, then, for every $\Sigma \supseteq \Theta_{\mathcal{M}}$, every $\Sigma$-expansion $\mathcal{N}^{\prime}$ of $\mathcal{N}$, and all $\alpha, \beta \in L(\Sigma)$, we have: 
(1) If $F\left(\phi_{\bar{w}_{n}}^{k}\right)=+$ (resp. $F\left(\phi_{\bar{w}_{n}}^{k}\right)=-$ ) and $\mathcal{N}^{\prime}, f\left(\bar{w}_{k}\right) \models_{\mathcal{L}} \phi_{\bar{w}_{n}}^{k}(\alpha, \beta)$ (resp. $\left.\mathcal{N}^{\prime}, f\left(\bar{w}_{k}\right) \forall_{\mathcal{L}} \phi_{\bar{w}_{n}}^{k}(\alpha, \beta)\right)$, then $\mathcal{N}^{\prime}, f\left(\bar{w}_{n}\right) \forall_{\mathcal{L}} \alpha \rightarrow \beta$.

(2) If $F\left(\psi_{\bar{w}_{n}}^{k}\right)=+$ (resp. $F\left(\psi_{\bar{w}_{n}}^{k}\right)=-$ ) and $\mathcal{N}^{\prime}, f\left(\bar{w}_{k}\right) \models_{\mathcal{L}} \psi_{\bar{w}_{n}}^{k}(\alpha, \beta)$ (resp. $\left.\mathcal{N}^{\prime}, f\left(\bar{w}_{k}\right) \forall_{\mathcal{L}} \psi_{\bar{w}_{n}}^{k}(\alpha, \beta)\right)$, then $\mathcal{N}^{\prime}, f\left(\bar{w}_{n}\right) \models_{\mathcal{L}} \alpha \ll \beta$.

(3) If $F\left(\theta_{\bar{w}_{n}}^{k}\right)=+$ (resp. $F\left(\theta_{\bar{w}_{n}}^{k}\right)=-$ ) and $\mathcal{N}^{\prime}, f\left(\bar{w}_{k}\right) \models_{\mathcal{L}} \theta_{\bar{w}_{n}}^{k}(\alpha, \beta)$ (resp. $\left.\mathcal{N}^{\prime}, f\left(\bar{w}_{k}\right) \not \nvdash_{\mathcal{L}} \theta_{\bar{w}_{n}}^{k}(\alpha, \beta)\right)$, then $\mathcal{N}^{\prime}, f\left(\bar{w}_{n}\right) \models_{\mathcal{L}} \alpha \rightarrow \beta$.

(4) If $F\left(\tau_{\bar{w}_{n}}^{k}\right)=+$ (resp. $F\left(\tau_{\bar{w}_{n}}^{k}\right)=-$ ) and $\mathcal{N}^{\prime}, f\left(\bar{w}_{k}\right) \models_{\mathcal{L}} \tau_{\bar{w}_{n}}^{k}(\alpha, \beta)$ (resp. $\left.\mathcal{N}^{\prime}, f\left(\bar{w}_{k}\right) \not \nvdash_{\mathcal{L}} \tau_{\bar{w}_{n}}^{k}(\alpha, \beta)\right)$, then $\mathcal{N}^{\prime}, f\left(\bar{w}_{n}\right) \nvdash_{\mathcal{L}} \alpha \ll \beta$.

Proof. We fix a $\bar{w}_{n} \in W_{w}^{u n}$ and argue by induction on $t:=n-k$ so that we have $0 \leq t \leq n-1$.

Induction Basis. $t=0$. Then $k=n$ and we set:

$$
\begin{array}{ll}
\phi_{\bar{w}_{n}}^{n}(\alpha, \beta):=\alpha \rightarrow \beta & F\left(\phi_{\bar{w}_{n}}^{n}\right)=- \\
\psi_{\bar{w}_{n}}^{n}(\alpha, \beta):=\alpha \ll \beta & F\left(\psi_{\bar{w}_{n}}^{n}\right)=+ \\
\theta_{\bar{w}_{n}}^{n}(\alpha, \beta):=\alpha \rightarrow \beta & F\left(\theta_{\bar{w}_{n}}^{n}\right)=+ \\
\tau_{\bar{w}_{n}}^{n}(\alpha, \beta):=\alpha \ll \beta & F\left(\tau_{\bar{w}_{n}}^{n}\right)=-
\end{array}
$$

It is evident that the statement of the Lemma holds under these settings.

Induction Step. Let $t=l+1$, so that $k=n-l-1$. Applying the Induction Hypothesis for $t=l$ with $k=n-l$, we find the formula schemas $\phi_{\bar{w}_{n}}^{n-l}, \psi_{\bar{w}_{n}}^{n-l}, \theta_{\bar{w}_{n}}^{n-l}$, and $\tau_{\bar{w}_{n}}^{n-l}$, and $F\left(\phi_{\bar{w}_{n}}^{n-l}\right), F\left(\psi_{\bar{w}_{n}}^{n-l}\right), F\left(\theta_{\bar{w}_{n}}^{n-l}\right)$, and $F\left(\tau_{\bar{w}_{n}}^{n-l}\right)$ satisfying the statement of the Lemma. We then have to distinguish between two cases.

Case 1. We have $w_{n-l-1} \prec w_{n-l}$, thus also $\bar{w}_{n-l-1} \prec_{w}^{u n} \bar{w}_{n-l}$. We then set $F\left(\phi_{\bar{w}_{n}}^{n-l-1}\right)=F\left(\psi_{\bar{w}_{n}}^{n-l-1}\right)=F\left(\theta_{\bar{w}_{n}}^{n-l-1}\right)=F\left(\tau_{\bar{w}_{n}}^{n-l-1}\right):=+$, and, as for the formulas, we set that

$$
\chi_{\bar{w}_{n}}^{n-l-1}(\alpha, \beta):= \begin{cases}q_{\bar{w}_{n-l}}^{+} \rightarrow \chi_{\bar{w}_{n}}^{n-l}(\alpha, \beta), & \text { if } F\left(\chi_{\bar{w}_{n}}^{n-l}\right)=+ \\ \chi_{\bar{w}_{n}}^{n-l}(\alpha, \beta) \rightarrow q_{\bar{w}_{n-l}}, & \text { if } F\left(\chi_{\bar{w}_{n}}^{n-l}\right)=-\end{cases}
$$

for every $\chi \in\{\phi, \psi, \theta, \tau\}$. We show that our settings satisfy the Lemma.

Case 1.1. Let $\chi \in\{\phi, \psi, \theta, \tau\}$ and assume that $F\left(\chi_{\bar{w}_{n}}^{n-l}\right)=+$. Then, if $\mathcal{N}^{\prime}, f\left(\bar{w}_{n-l-1}\right) \models_{\mathcal{L}} q_{\bar{w}_{n-l}}^{+} \rightarrow \chi_{\bar{w}_{n}}^{n-l}(\alpha, \beta)$, note that we must have both $f\left(\bar{w}_{n-l-1}\right) \triangleleft f\left(\bar{w}_{n-l}\right)$, by $\bar{w}_{n-l-1} \prec_{w}^{u n} \bar{w}_{n-l}$ and condition

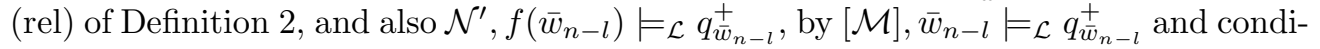
tion (theories) of Definition 2. Therefore, we must also have $\mathcal{N}^{\prime}, f\left(\bar{w}_{n-l}\right) \models_{\mathcal{L}} \chi_{\bar{w}_{n}}^{n-l}(\alpha, \beta)$, so that we can apply Induction Hypothesis for $l$.

Case 1.2. Let $\chi \in\{\phi, \psi, \theta, \tau\}$ and assume that $F\left(\chi_{\bar{w}_{n}}^{n-l}\right)=-$. Then, if $\mathcal{N}^{\prime}, f\left(\bar{w}_{n-l-1}\right) \models_{\mathcal{L}} \chi_{\bar{w}_{n}}^{n-l}(\alpha, \beta) \rightarrow q_{\bar{w}_{n-l}}^{\bar{w}}$, note that we must have both $f\left(\bar{w}_{n-l-1}\right) \triangleleft f\left(\bar{w}_{n-l}\right)$, by $\bar{w}_{n-l-1} \prec_{w}^{u n} \bar{w}_{n-l}$ and condition

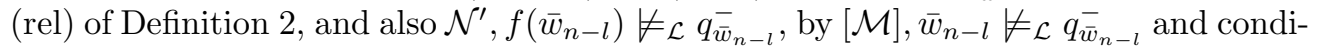
tion (theories) of Definition 2. Therefore, we must also have $\mathcal{N}^{\prime}, f\left(\bar{w}_{n-l}\right) \not \nvdash_{\mathcal{L}} \chi_{\bar{w}_{n}}^{n-l}(\alpha, \beta)$, so that we can apply Induction Hypothesis for $l$.

Case 2. We have $w_{n-l-1} \succ w_{n-l}$, thus also $\bar{w}_{n-l} \prec_{w}^{u n} \bar{w}_{n-l-1}$. We then set $F\left(\phi_{\bar{w}_{n}}^{n-l-1}\right)=F\left(\psi_{\bar{w}_{n}}^{n-l-1}\right)=F\left(\theta_{\bar{w}_{n}}^{n-l-1}\right)=F\left(\tau_{\bar{w}_{n}}^{n-l-1}\right):=-$, and, as for the formulas, we set that

$$
\chi_{\bar{w}_{n}}^{n-l-1}(\alpha, \beta):=\left\{\begin{array}{l}
q_{\bar{w}_{n-l}}^{+} \ll \chi_{\bar{w}_{n}}^{n-l}(\alpha, \beta), \text { if } F\left(\chi_{\bar{w}_{n}}^{n-l}\right)=+ \\
\chi_{\bar{w}_{n}}^{n-l}(\alpha, \beta) \ll q_{\bar{w}_{n-l}}, \text { if } F\left(\chi_{\bar{w}_{n}}^{n-l}\right)=-
\end{array}\right.
$$

for every $\chi \in\{\phi, \psi, \theta, \tau\}$. We show that our settings satisfy the Lemma. 
Case 2.1. Let $\chi \in\{\phi, \psi, \theta, \tau\}$ and assume that $F\left(\chi_{\bar{w}_{n}}^{n-l}\right)=+$. Then, if $\mathcal{N}^{\prime}, f\left(\bar{w}_{n-l-1}\right) \nvdash_{\mathcal{L}} q_{\bar{w}_{n-l}}^{+} \ll \chi_{\bar{w}_{n}}^{n-l}(\alpha, \beta)$, note that we must have both $f\left(\bar{w}_{n-l}\right) \triangleleft f\left(\bar{w}_{n-l-1}\right)$, by $\bar{w}_{n-l} \prec_{w}^{u n} \bar{w}_{n-l-1}$ and condition (rel) of Definition 2, and also $\mathcal{N}^{\prime}, f\left(\bar{w}_{n-l}\right) \models_{\mathcal{L}} q_{\bar{w}_{n-l}}^{+}$, by $[\mathcal{M}], \bar{w}_{n-l} \models_{\mathcal{L}} q_{\bar{w}_{n-l}}^{+}$and condition (theories) of Definition 2. Therefore, we must also have $\mathcal{N}^{\prime}, f\left(\bar{w}_{n-l}\right) \models_{\mathcal{L}} \chi_{\bar{w}_{n}^{n}}^{n-l}(\alpha, \beta)$, so that we can apply Induction Hypothesis for $l$.

Case 2.2. Let $\chi \in\{\phi, \psi, \theta, \tau\}$ and assume that $F\left(\chi_{\bar{w}_{n}}^{n-l}\right)=-$. Then, if $\mathcal{N}^{\prime}, f\left(\bar{w}_{n-l-1}\right) \not \nvdash_{\mathcal{L}} \chi_{\bar{w}_{n}}^{n-l}(\alpha, \beta) \ll q_{\bar{w}_{n-l}}^{\bar{w}_{n}}$, note that we must have both $f\left(\bar{w}_{n-l}\right) \triangleleft f\left(\bar{w}_{n-l-1}\right)$, by $\bar{w}_{n-l} \prec_{w}^{u n} \bar{w}_{n-l-1}$ and condition

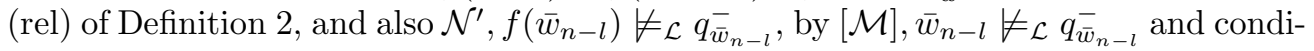
tion (theories) of Definition 2. Therefore, we must also have $\mathcal{N}^{\prime}, f\left(\bar{w}_{n-l}\right) \nvdash_{\mathcal{L}} \chi_{\bar{w}_{n}}^{n-l}(\alpha, \beta)$ so that we can apply Induction Hypothesis for $l$.

Furthermore, if in the above Lemma we replace $\mathcal{N}$ with $[\mathcal{M}]$, then the conditionals in the lemma can be strengthened to bi-conditionals:

Lemma 12. Let $\mathcal{L}$ be an abstract bi-intuitionistic logic which extends $\mathrm{BIL}$, is preserved under bi-asimulations, $\star$-compact, and has TUP, and let $(\mathcal{M}, w) \in \operatorname{Pmod}_{\Theta}$ be a biunravelled model. Then, for every $n>0$, every $\bar{w}_{n} \in W_{w}^{u n}$, and every $1 \leq k \leq n$ let the binary formula schemas $\phi_{\bar{w}_{n}}^{k}\left(\__{1}, \__{2}\right), \psi_{\bar{w}_{n}}^{k}\left(-_{1}, \__{2}\right), \theta_{\bar{w}_{n}}^{k}\left(\__{1}, \__{2}\right), \tau_{\bar{w}_{n}}^{k}\left(-_{1}, \__{2}\right) \in B I L\left(\Theta_{\mathcal{M}}\right)$, and the function

$$
F:\left\{\phi_{\bar{w}_{n}}^{k}, \psi_{\bar{w}_{n}}^{k}, \theta_{\bar{w}_{n}}^{k}, \tau_{\bar{w}_{n}}^{k} \mid \bar{w}_{n} \in W_{w}^{u n}, 1 \leq k \leq n\right\} \rightarrow\{1,-1\}
$$

be defined as in Lemma 11. Then, for every $\Sigma \supseteq \Theta_{\mathcal{M}}$, every $\Sigma$-expansion $\mathcal{M}^{\prime}$ of $[\mathcal{M}]$, and all $\alpha, \beta \in L(\Sigma)$, we have:

(1) If $F\left(\phi_{\bar{w}_{n}}^{k}\right)=+$ (resp. $F\left(\phi_{\bar{w}_{n}}^{k}\right)=-$ ) then $\mathcal{M}^{\prime}, \bar{w}_{k} \models_{\mathcal{L}} \phi_{\bar{w}_{n}}^{k}(\alpha, \beta)\left(\right.$ resp. $\mathcal{M}^{\prime}, \bar{w}_{k} \nvdash_{\mathcal{L}} \phi_{\bar{w}_{n}}^{k}(\alpha, \beta)$ ) iff $\mathcal{M}^{\prime}, \bar{w}_{n} \not \neq_{\mathcal{L}} \alpha \rightarrow \beta$.

(2) If $F\left(\psi_{\bar{w}_{n}}^{k}\right)=+$ (resp. $F\left(\psi_{\bar{w}_{n}}^{k}\right)=-$ ), then $\mathcal{M}^{\prime}, \bar{w}_{k} \models_{\mathcal{L}} \psi_{\bar{w}_{n}}^{k}(\alpha, \beta)$ (resp. $\left.\mathcal{M}^{\prime}, \bar{w}_{k} \nvdash_{\mathcal{L}} \psi_{\bar{w}_{n}}^{k}(\alpha, \beta)\right)$ iff $\mathcal{M}^{\prime}, \bar{w}_{n} \models_{\mathcal{L}} \alpha \ll \beta$.

(3) If $F\left(\theta_{\bar{w}_{n}}^{k}\right)=+$ (resp. $\left.F\left(\theta_{\bar{w}_{n}}^{k}\right)=-\right)$, then $\mathcal{M}^{\prime}, \bar{w}_{k} \models_{\mathcal{L}} \theta_{\bar{w}_{n}}^{k}(\alpha, \beta)\left(\right.$ resp. $\left.\mathcal{N}^{\prime}, \bar{w}_{k} \nvdash_{\mathcal{L}} \theta_{\bar{w}_{n}}^{k}(\alpha, \beta)\right)$ iff $\mathcal{M}^{\prime}, \bar{w}_{n} \models_{\mathcal{L}} \alpha \rightarrow \beta$.

(4) If $F\left(\tau_{\bar{w}_{n}}^{k}\right)=+\left(\right.$ resp. $\left.F\left(\tau_{\bar{w}_{n}}^{k}\right)=-\right)$, then $\mathcal{M}^{\prime}, \bar{w}_{k} \models_{\mathcal{L}} \tau_{\bar{w}_{n}}^{k}(\alpha, \beta)\left(\right.$ resp. $\left.\mathcal{M}^{\prime}, \bar{w}_{k} \nvdash_{\mathcal{L}} \tau_{\bar{w}_{n}}^{k}(\alpha, \beta)\right)$ iff $\mathcal{M}^{\prime}, \bar{w}_{n} \not \forall_{\mathcal{L}} \alpha \ll \beta$.

Proof. Since $i d_{W_{w}^{u n}}$ is, by the Isomorphism Property, an $\mathcal{L}$-elementary embedding of $[\mathcal{M}]$ into itself, we have the $(\Rightarrow)$-part of the Lemma by Lemma 11. As for the $(\Leftarrow)$ part, we, again, fix a $\bar{w}_{n} \in W_{w}^{u n}$ and argue by induction on $t:=n-k$ so that we have $0 \leq t \leq n-1$. Induction basis is obvious, given the definitions in the proof of Lemma 11.

Induction Step. Let $t=l+1$, so that $k=n-l-1$. Then Induction Hypothesis for $t=l$ with $k=n-l$, tells us that the formulas $\phi_{\bar{w}_{n}}^{n-l}, \psi_{\bar{w}_{n}}^{n-l}, \theta_{\bar{w}_{n}}^{n-l}$, and $\tau_{\bar{w}_{n}}^{n-l}$, as well as $F\left(\phi_{\bar{w}_{n}}^{n-l}\right), F\left(\psi_{\bar{w}_{n}}^{n-l}\right), F\left(\theta_{\bar{w}_{n}}^{n-l}\right)$, and $F\left(\tau_{\bar{w}_{n}}^{n-l}\right)$ satisfy the statement of the Lemma. We then have to distinguish between two cases.

Case 1. We have $w_{n-l-1} \prec w_{n-l}$, thus also $\bar{w}_{n-l-1} \prec_{w}^{u n} \bar{w}_{n-l}$. We then have that $F\left(\phi_{\bar{w}_{n}}^{n-l-1}\right)=F\left(\psi_{\bar{w}_{n}}^{n-l-1}\right)=F\left(\theta_{\bar{w}_{n}}^{n-l-1}\right)=F\left(\tau_{\bar{w}_{n}}^{n-l-1}\right)=+$, and, as for the formulas, that

$$
\chi_{\bar{w}_{n}}^{n-l-1}(\alpha, \beta)= \begin{cases}q_{\bar{w}_{n-l}}^{+} \rightarrow \chi_{\bar{w}_{n}}^{n-l}(\alpha, \beta), & \text { if } F\left(\chi_{\bar{w}_{n}}^{n-l}\right)=+ \\ \chi_{\bar{w}_{n}}^{n-l}(\alpha, \beta) \rightarrow q_{\bar{w}_{n-l}}, & \text { if } F\left(\chi_{\bar{w}_{n}}^{n-l}\right)=-\end{cases}
$$


for every $\chi \in\{\phi, \psi, \theta, \tau\}$. We show that these settings satisfy the $(\Leftarrow)$-part of the Lemma.

Case 1.1. Let $\chi \in\{\phi, \psi, \theta, \tau\}$ and assume that $F\left(\chi_{\bar{w}_{n}}^{n-l}\right)=+$. Assume that the condition associated with $\chi_{\bar{w}_{n}}^{n-l-1}(\alpha, \beta)$ by the Lemma, holds in $\mathcal{M}^{\prime}$. Then, by Induction Hypothesis, we must have $\mathcal{M}^{\prime}, \bar{w}_{n-l} \models_{\mathcal{L}} \chi_{\bar{w}_{n}^{n}}^{n-l}(\alpha, \beta)$. Now, let $v \in W_{w}^{u n}$ be such that $\bar{w}_{n-l-1} \prec_{w}^{u n} v$ and $\mathcal{M}^{\prime}, v \models_{\mathcal{L}} q_{\bar{w}_{n-l}}^{\bar{w}_{n-l}}$. By the definition of $[\mathcal{M}]$, it follows that $\bar{w}_{n-l} \prec_{w}^{u n} v$, but then we must also have $\mathcal{M}^{\prime}, v \models_{\mathcal{L}} \chi_{\bar{w}_{n}}^{n-l}(\alpha, \beta)$, by Lemma 8 ,

Case 1.2. Let $\chi \in\{\phi, \psi, \theta, \tau\}$ and assume that $F\left(\chi_{\bar{w}_{n}}^{n-l}\right)=-$. Assume that the condition associated with $\chi_{\bar{w}_{n}}^{n-l-1}(\alpha, \beta)$ by the Lemma, holds in $\mathcal{M}^{\prime}$. Then, by Induction Hypothesis, we must have $\mathcal{M}^{\prime}, \bar{w}_{n-l} \nvdash_{\mathcal{L}} \chi_{\bar{w}_{n}^{n}}^{n-l}(\alpha, \beta)$. Now, let $v \in W_{w}^{u n}$ be such that $\bar{w}_{n-l-1} \prec_{w}^{u n} v$ and $\mathcal{M}^{\prime}, v \nvdash_{\mathcal{L}} q_{\bar{w}_{n-l}}^{\bar{w}_{n-l}}$. By the definition of $[\mathcal{M}]$, it follows that $v \prec_{w}^{u n} \bar{w}_{n-l}$, but then we must also have $\mathcal{M}^{\prime}, v \nvdash_{\mathcal{L}} \chi_{\bar{w}_{n}}^{n-l}(\alpha, \beta)$, by Lemma 8 ,

Case 2. We have $w_{n-l-1} \succ w_{n-l}$, thus also $\bar{w}_{n-l} \prec_{w}^{\text {un }} \bar{w}_{n-l-1}$. We have then that $F\left(\phi_{\bar{w}_{n}}^{n-l-1}\right)=F\left(\psi_{\bar{w}_{n}}^{n-l-1}\right)=F\left(\theta_{\bar{w}_{n}}^{n-l-1}\right)=F\left(\tau_{\bar{w}_{n}}^{n-l-1}\right):=-$, and, as for the formulas, that

$$
\chi_{\bar{w}_{n}}^{n-l-1}(\alpha, \beta):= \begin{cases}q_{\bar{w}_{n-l}}^{+} \ll \chi_{\bar{w}_{n}}^{n-l}(\alpha, \beta), & \text { if } F\left(\chi_{\bar{w}_{n}}^{n-l}\right)=+ \\ \chi_{\bar{w}_{n}}^{n-l}(\alpha, \beta) \ll q_{\bar{w}_{n-l}}, & \text { if } F\left(\chi_{\bar{w}_{n}}^{n-l}\right)=-\end{cases}
$$

for every $\chi \in\{\phi, \psi, \theta, \tau\}$. We show that these settings satisfy the $(\Leftarrow)$-part of the Lemma.

Case 2.1. Let $\chi \in\{\phi, \psi, \theta, \tau\}$ and assume that $F\left(\chi_{\bar{w}_{n}}^{n-l}\right)=+$. Assume that the condition associated with $\chi_{\bar{w}_{n}}^{n-l-1}(\alpha, \beta)$ by the Lemma, holds in $\mathcal{M}^{\prime}$. Then, by Induction Hypothesis, we must have $\mathcal{M}^{\prime}, \bar{w}_{n-l} \models_{\mathcal{L}} \chi_{\bar{w}_{n}^{n}}^{n-l}(\alpha, \beta)$. Now, let $v \in W_{w}^{u n}$ be such that $v \prec_{w}^{u n} \bar{w}_{n-l-1}$ and $\mathcal{M}^{\prime}, v \models_{\mathcal{L}} q_{\bar{w}_{n-l}}^{+}$. By the definition of $[\mathcal{M}]$, it follows that $\bar{w}_{n-l} \prec_{w}^{u n} v$, but then we must also have $\mathcal{M}^{\prime}, v \models_{\mathcal{L}} \chi_{\bar{w}_{n}}^{n-l}(\alpha, \beta)$, by Lemma 8 ,

Case 2.2. Let $\chi \in\{\phi, \psi, \theta, \tau\}$ and assume that $F\left(\chi_{\bar{w}_{n}}^{n-l}\right)=-$. Assume that the condition associated with $\chi_{\bar{w}_{n}}^{n-l-1}(\alpha, \beta)$ by the Lemma, holds in $\mathcal{M}^{\prime}$. Then, by Induction Hypothesis, we must have $\mathcal{M}^{\prime}, \bar{w}_{n-l} \nvdash_{\mathcal{L}} \chi_{\bar{w}_{n}^{n}}^{n-l}(\alpha, \beta)$. Now, let $v \in W_{w}^{u n}$ be such

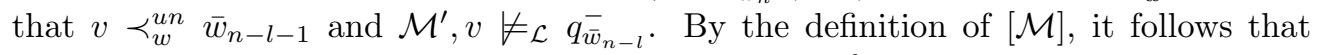
$v \prec_{w}^{u n} \bar{w}_{n-l}$, but then we must also have $\mathcal{M}^{\prime}, v \nvdash_{\mathcal{L}} \chi_{\bar{w}_{n}}^{n-l}(\alpha, \beta)$, by Lemma 8 ,

Proposition 2. Let $\mathcal{L}$ be an abstract bi-intuitionistic logic which extends $\mathrm{BIL}$, is preserved under bi-asimulations, $\star$-compact, and has TUP, and let $(\mathcal{M}, w) \in \operatorname{Pmod}_{\Theta}$ be a bi-unravelled model. Then there exists a bi-unravelled model $\left(\mathcal{N}^{\prime}, w\right) \in \operatorname{Pmod}_{\Theta}$ such that $\mathcal{M} \preccurlyeq \mathcal{L} \mathcal{N}^{\prime}$ and every $\mathcal{L}$-type of $\mathcal{M}$ is realized in $\mathcal{N}^{\prime}$.

Proof. Assume the hypothesis of the Proposition. For the given $\mathcal{M}$, consider $[\mathcal{M}]$; we extend the signature of this model as follows:

- For every $\bar{v}_{k} \in W_{w}^{u n}$, if $\Gamma, \Delta \subseteq L\left(\Theta_{\mathcal{M}}\right)$ are such that $(\Gamma, \Delta)$ is a successor $\mathcal{L}$-type of $\left([\mathcal{M}], \bar{v}_{k}\right)$, then we add two fresh propositional letters $r_{\Gamma, \Delta, \bar{v}_{k}}^{+}$and $r_{\Gamma, \Delta, \bar{v}_{k}}^{-}$.

- For every $\bar{v}_{k} \in W_{w}^{u n}$, if $\Gamma, \Delta \subseteq L\left(\Theta_{\mathcal{M}}\right)$ are such that $(\Gamma, \Delta)$ is a predecessor $\mathcal{L}$-type of $\left([\mathcal{M}], \bar{v}_{k}\right)$, then we add two fresh propositional letters $s_{\Gamma, \Delta, \bar{v}_{k}}^{+}$and $s_{\Gamma, \Delta, \bar{v}_{k}}^{-} \cdot$ 
We will call the resulting signature $\Theta^{\prime}$. Consider, next, the following set of formulas:

$$
\begin{aligned}
\Upsilon=\left\{\phi_{\bar{v}_{k}}^{1}\left(r_{\Gamma, \Delta, \bar{v}_{k}}^{+}, r_{\Gamma, \Delta, \bar{v}_{k}}^{-}\right), \theta_{\bar{v}_{k}}\left(r_{\Gamma, \Delta, \bar{v}_{k}}^{+}, \gamma\right), \theta_{\bar{v}_{k}}^{1}\left(\delta, r_{\Gamma, \Delta, \bar{v}_{k}}^{-}\right) \mid r_{\Gamma, \Delta, \bar{v}_{k}}^{+}, r_{\Gamma, \Delta, \bar{v}_{k}}^{-} \in \Theta^{\prime}, \gamma \in \Gamma, \delta \in \Delta\right\} \cup \\
\cup\left\{\psi_{\bar{v}_{k}}^{1}\left(s_{\Gamma, \Delta, \bar{v}_{k}}^{+}, s_{\Gamma, \Delta, \bar{v}_{k}}^{-}\right), \tau_{\bar{v}_{k}}\left(s_{\Gamma, \Delta, \bar{v}_{k}}^{+}, \gamma\right), \tau_{\bar{v}_{k}}^{1}\left(\delta, s_{\Gamma, \Delta, \bar{v}_{k}}^{-}\right) \mid s_{\Gamma, \Delta, \bar{v}_{k}}^{+}, s_{\Gamma, \Delta, \bar{v}_{k}}^{-} \in \Theta^{\prime}, \gamma \in \Gamma, \delta \in \Delta\right\}
\end{aligned}
$$

We partition $\Upsilon$ into two subset: $\left\{\right.$, $\Upsilon^{+}=\{\eta \in \Upsilon \mid F(\eta)=+\}$ and $\Upsilon^{-}=\{\eta \in \Upsilon \mid F(\eta)=-\}$, and consider the following $L\left(\Theta^{\prime}\right)$-theory $\Xi=\left(\Xi^{+}, \Xi^{-}\right)=\left(T h_{\mathcal{L}}^{+}([\mathcal{M}], w) \cup \Upsilon^{+}, T h_{\mathcal{L}}^{-}([\mathcal{M}], w) \cup \Upsilon^{-}\right)$.

We show that $\Xi$ is finitely $\mathcal{L}$-satisfiable. Indeed, given an arbitrary $\left(S_{0}, T_{0}\right) \Subset \Xi$ we know, wlog, that, for some $n<\omega$ we have:

$$
\left(S_{0}, T_{0}\right)=\left(S_{1}, T_{1}\right) \cup \ldots \cup\left(S_{n}, T_{n}\right),
$$

where for every $1 \leq i \leq n$ one of the following two cases holds:

Case 1. For some $\bar{v}_{k} \in W_{w}^{u n}$, there exist $\Gamma, \Delta \subseteq L\left(\Theta_{\mathcal{M}}\right)$ such that $(\Gamma, \Delta)$ is a successor $\mathcal{L}$-type of $\left([\mathcal{M}], \bar{v}_{k}\right)$ and $\Gamma^{\prime} \Subset \Gamma, \Delta^{\prime} \Subset \Delta$ such that we have: $\left(S_{i}, T_{i}\right)=\left(S_{i}^{\prime} \cup S_{i}^{\prime \prime}, T_{i}^{\prime} \cup T_{i}^{\prime \prime}\right)$, where $\left(S_{i}^{\prime \prime}, T_{i}^{\prime \prime}\right) \Subset\left(T h_{\mathcal{L}}([\mathcal{M}], w)\right.$ and:

$$
S_{i}^{\prime} \cup T_{i}^{\prime} \subseteq\left\{\phi_{\bar{v}_{k}}^{1}\left(r_{\Gamma, \Delta, \bar{v}_{k}}^{+}, r_{\Gamma, \Delta, \bar{v}_{k}}^{-}\right), \theta_{\bar{v}_{k}}^{1}\left(r_{\Gamma, \Delta, \bar{v}_{k}}^{+}, \gamma\right), \theta_{\bar{v}_{k}}^{1}\left(\delta, r_{\Gamma, \Delta, \bar{v}_{k}}^{-}\right) \mid \gamma \in \Gamma^{\prime}, \delta \in \Delta^{\prime}\right\}
$$

By Definition [3 $\left(\Gamma^{\prime}, \Delta^{\prime}\right)$ is $\mathcal{L}$-satisfied at $\left([\mathcal{M}], \bar{u}_{m}\right)$ for some $\bar{u}_{m} \in W_{w}^{u n}$ such that $\bar{v}_{k} \prec_{w}^{u n} \bar{u}_{m}$. But then it follows from Lemma 12, that $\left(S_{i}, T_{i}\right)$ must be $\mathcal{L}$-satisfied at $w$ in the expansion $\mathcal{M}^{\prime}$ of $[\mathcal{M}]$ in which $r_{\Gamma, \Delta, \bar{v}_{k}}^{+}$and $r_{\Gamma, \Delta, \bar{v}_{k}}^{-}$are identified with $q_{\bar{u}_{m}}^{+}, q_{\bar{u}_{m}}^{-}$, respectively.

Case 2. For some $\bar{v}_{k} \in W_{w}^{u n}$, there exist $\Gamma, \Delta \subseteq L\left(\Theta_{\mathcal{M}}\right)$ such that $(\Gamma, \Delta)$ is a predecessor $\mathcal{L}$-type of $\left([\mathcal{M}], \bar{v}_{k}\right)$ and $\Gamma^{\prime} \Subset \Gamma, \Delta^{\prime} \Subset \Delta$ such that we have: $\left(S_{i}, T_{i}\right)=\left(S_{i}^{\prime} \cup S_{i}^{\prime \prime}, T_{i}^{\prime} \cup T_{i}^{\prime \prime}\right)$, where $\left(S_{i}^{\prime \prime}, T_{i}^{\prime \prime}\right) \Subset\left(T h_{\mathcal{L}}([\mathcal{M}], w)\right.$ and:

$$
S_{i}^{\prime} \cup T_{i}^{\prime} \subseteq\left\{\psi_{\bar{v}_{k}}^{1}\left(s_{\Gamma, \Delta, \bar{v}_{k}}^{+}, s_{\Gamma, \Delta, \bar{v}_{k}}^{-}\right), \tau_{\bar{v}_{k}}^{1}\left(s_{\Gamma, \Delta, \bar{v}_{k}}^{+}, \gamma\right), \tau_{\bar{v}_{k}}^{1}\left(\delta, s_{\Gamma, \Delta, \bar{v}_{k}}^{-}\right) \mid \gamma \in \Gamma^{\prime}, \delta \in \Delta^{\prime}\right\}
$$

By Definition $3\left(\Gamma^{\prime}, \Delta^{\prime}\right)$ is $\mathcal{L}$-satisfied at $\left([\mathcal{M}], \bar{u}_{m}\right)$ for some $\bar{u}_{m} \in W_{w}^{u n}$ such that $\bar{u}_{m} \prec_{w}^{u n} \bar{v}_{k}$. But then it follows from Lemma 12, that $\left(S_{i}, T_{i}\right)$ must be $\mathcal{L}$-satisfied at $w$ in the expansion $\mathcal{M}^{\prime}$ of $[\mathcal{M}]$ in which $s_{\Gamma, \Delta, \bar{v}_{k}}^{+}$and $s_{\Gamma, \Delta, \bar{v}_{k}}^{-}$are identified with $q_{\bar{u}_{m}}^{+}, q_{\bar{u}_{m}}^{-}$, respectively.

Note, moreover, that for all $1 \leq i<j \leq n$, the set of propositional letters that needs to be added to $[\mathcal{M}]$ in order to get $T_{i}$ satisfied at $w$ is disjoint from the set of propositional letters to be added to this same model in order to get $T_{j}$ satisfied at $w$. Therefore, we can take the union of the expansions required by $T_{1}, \ldots, T_{n}$ and get (by Expansion property) an expansion $\mathcal{M}^{\prime}$ of $[\mathcal{M}]$ such that $\left(S_{0}, T_{0}\right)$ is satisfied at $\left(\mathcal{M}^{\prime}, w\right)$. Since $\left(S_{0}, T_{0}\right) \Subset \Xi$ was chosen arbitrarily, this means, by the $\star$-compactness of $\mathcal{L}$, that $\Xi$ itself is $\mathcal{L}$-satisfiable.

Let $\left(\mathcal{M}_{1}, w_{1}\right)$ be a pointed $\Theta^{\prime}$-model $\mathcal{L}$-satisfying $\Xi$. We know, by Lemma 7 and the preservation of $\mathcal{L}$ under bi-asimulations, that $\left(\left(\mathcal{M}_{1}\right)_{w_{1}}^{u n}, w_{1}\right)$ also $\mathcal{L}$-satisfies $\Xi$. We now set $\mathcal{M}^{\prime}:=\left(\mathcal{M}_{1}\right)_{w_{1}}^{u n}\left\lceil\Theta_{\mathcal{M}}\right.$. It follows, by Expansion Property, that $\mathcal{M}^{\prime} \mathcal{L}$-satisfies $T h_{\mathcal{L}}([\mathcal{M}], w)$. Therefore, by Lemma 10 there must be an $\mathcal{L}$-elementary embedding $f$ of $[\mathcal{M}]$ into $\mathcal{M}^{\prime}$, and for this elementary embedding we will have with $f(w)=w_{1}$. Note, moreover, that, by Lemma 1,5, we have then $f([\mathcal{M}]) \preccurlyeq_{\mathcal{L}} \mathcal{M}^{\prime}$ where $f([\mathcal{M}])$ is an $f$-isomorphic copy of $[\mathcal{M}]$. Therefore, by Lemma 1]6, there must exist a $\Theta_{\mathcal{M}}$-model $\mathcal{N}$ and a function $f^{\prime} \supseteq f$, such that $[\mathcal{M}] \preccurlyeq \mathcal{L} \mathcal{N}$, and $f^{\prime}: \mathcal{N} \cong \mathcal{M}^{\prime}$. We now prove the following:

Claim. $\mathcal{N}$ realizes every $\mathcal{L}$-type of $[\mathcal{M}]$.

\footnotetext{
${ }^{4}$ Throughout this proof, we set that $F(\alpha):=F(\chi)$ if $\alpha$ is a substitution case of $\chi$.
} 
To prove this Claim, we again have to consider the two cases outlined above.

Case 1. For some $\bar{u}_{r} \in W_{w}^{u n}$, there exist $\Gamma, \Delta \subseteq L\left(\Theta_{\mathcal{M}}\right)$ such that $(\Gamma, \Delta)$ is a successor $\mathcal{L}$-type of $\left([\mathcal{M}], \bar{u}_{r}\right)$. Note that for every formula $\chi$ in the set:

$$
\left\{\phi_{\bar{u}_{r}}^{1}\left(r_{\Gamma, \Delta, \bar{u}_{r}}^{+}, r_{\Gamma, \Delta, \bar{u}_{r}}^{-}\right), \theta_{\bar{u}_{r}}^{1}\left(r_{\Gamma, \Delta, \bar{u}_{r}}^{+}, \gamma\right), \theta_{\bar{u}_{r}}^{1}\left(\delta, r_{\Gamma, \Delta, \bar{u}_{r}}^{-}\right) \mid \gamma \in \Gamma, \delta \in \Delta\right\}
$$

we have that $\left(\mathcal{M}_{1}\right)_{w_{1}}^{u n}, w_{1} \models_{\mathcal{L}} \chi$ iff $F(\chi)=+$. Therefore, since we also have $w_{1}=f(w)=f\left(u_{1}\right)$ (by $\bar{u}_{r} \in W_{w}^{u n}$ and the definition of bi-unravelling), it follows from Lemma 11 that for some $v \in\left(W_{1}\right)_{w_{1}}^{u n}$ we have both $f\left(\bar{u}_{r}\right)\left(\prec_{1}\right)_{w_{1}}^{u n} v$ and $\left(\mathcal{M}_{1}\right)_{w_{1}}^{u n}, v \models_{\mathcal{L}}\left(\left\{r_{\Gamma, \Delta, \bar{u}_{r}}^{+}\right\},\left\{r_{\Gamma, \Delta, \bar{u}_{r}}^{-}\right\}\right)$. On the other hand, it follows from the same Lemma 11 that for all $\gamma \in \Gamma$ and $\delta \in \Delta$ we have:

$$
\left(\mathcal{M}_{1}\right)_{w_{1}}^{u n}, f\left(\bar{u}_{r}\right) \models_{\mathcal{L}}\left(r_{\Gamma, \Delta, \bar{u}_{r}}^{+} \rightarrow \gamma\right) \wedge\left(\delta \rightarrow r_{\Gamma, \Delta, \bar{u}_{r}}^{-}\right) .
$$

But then, it follows, by the choice of $v$, that we have:

$$
\left(\mathcal{M}_{1}\right)_{w_{1}}^{u n}, v \models_{\mathcal{L}}(\Gamma, \Delta) .
$$

Since we have $\mathcal{M}^{\prime}=\left(\mathcal{M}_{1}\right)_{w_{1}}^{u n} \uparrow \Theta_{\mathcal{M}}$, it follows that also $f\left(\bar{u}_{r}\right) \prec^{\prime} v$, and, by Expansion Property, that:

$$
\mathcal{M}^{\prime}, v \models_{\mathcal{L}}(\Gamma, \Delta) .
$$

But then consider $u \in U$ such that $f^{\prime}(u)=v$. We have then $\bar{u}_{r} \triangleleft u$, and, by Lemma 14, that

so that $\mathcal{N}$ realizes $(\Gamma, \Delta)$.

$$
\mathcal{N}, u \models_{\mathcal{L}}(\Gamma, \Delta),
$$

Case 2. For some $\bar{u}_{r} \in W_{w}^{u n}$, there exist $\Gamma, \Delta \subseteq L\left(\Theta_{\mathcal{M}}\right)$ such that $(\Gamma, \Delta)$ is a predecessor $\mathcal{L}$-type of $\left([\mathcal{M}], \bar{u}_{r}\right)$. Note that for every formula $\chi$ in the set:

$$
\left\{\psi_{\bar{u}_{r}}^{1}\left(s_{\Gamma, \Delta, \bar{u}_{r}}^{+}, s_{\Gamma, \Delta, \bar{u}_{r}}^{-}\right), \tau_{\bar{u}_{r}}^{1}\left(s_{\Gamma, \Delta, \bar{u}_{r}}^{+}, \gamma\right), \tau_{\bar{u}_{r}}^{1}\left(\delta, s_{\Gamma, \Delta, \bar{u}_{r}}^{-}\right) \mid \gamma \in \Gamma, \delta \in \Delta\right\}
$$

we have that $\left(\mathcal{M}_{1}\right)_{w_{1}}^{u n}, w_{1} \models_{\mathcal{L}} \chi$ iff $F(\chi)=+$. Therefore, since we also have $w_{1}=f(w)=f\left(u_{1}\right)$ (by $\bar{u}_{r} \in W_{w}^{u n}$ and the definition of bi-unravelling), it follows from Lemma 11 that for some $v \in\left(W_{1}\right)_{w_{1}}^{u n}$ we have both $v\left(\prec_{1}\right)_{w_{1}}^{u n} f\left(\bar{u}_{r}\right)$ and $\left(\mathcal{M}_{1}\right)_{w_{1}}^{u n}, v \models_{\mathcal{L}}\left(\left\{s_{\Gamma, \Delta, \bar{u}_{r}}^{+}\right\},\left\{s_{\Gamma, \Delta, \bar{u}_{r}}^{-}\right\}\right)$. On the other hand, it follows from the same Lemma 11 that for all $\gamma \in \Gamma$ and $\delta \in \Delta$ we have:

$$
\left(\mathcal{M}_{1}\right)_{w_{1}}^{u n}, f\left(\bar{u}_{r}\right) \not \nvdash_{\mathcal{L}}\left(s_{\Gamma, \Delta, \bar{u}_{r}}^{+} \ll \gamma\right) \vee\left(\delta \ll s_{\Gamma, \Delta, \bar{u}_{r}}^{-}\right) .
$$

But then, it follows, by the choice of $v$, that we have:

$$
\left(\mathcal{M}_{1}\right)_{w_{1}}^{u n}, v \models_{\mathcal{L}}(\Gamma, \Delta) .
$$

Since we have $\mathcal{M}^{\prime}=\left(\mathcal{M}_{1}\right)_{w_{1}}^{u n}\left\lceil\Theta_{\mathcal{M}}\right.$, it follows that also $v \prec^{\prime} f\left(\bar{u}_{r}\right)$, and, by Expansion Property, that:

$$
\mathcal{M}^{\prime}, v \models_{\mathcal{L}}(\Gamma, \Delta) .
$$

But then consider $u \in U$ such that $f^{\prime}(u)=v$. We have then $u \triangleleft \bar{u}_{r}$, and, by Lemma 1.4, that

$$
\mathcal{N}, u \models_{\mathcal{L}}(\Gamma, \Delta),
$$

so that $\mathcal{N}$ realizes $(\Gamma, \Delta)$.

Our Claim is thus proven.

It remains to notice that, by Lemma $2,2, \mathcal{N}^{\prime}:=\mathcal{N} \uparrow \Theta$ must realize every $\mathcal{L}$-type of $\mathcal{M}=[\mathcal{M}]\left\lceil\Theta\right.$, and that, by $f^{\prime}: \mathcal{N} \cong \mathcal{M}^{\prime}, \mathcal{N}^{\prime}$ is a $\Theta$-reduct of a bi-unravelled model and hence a bi-unravelled $\Theta$-model itself. 
Corollary 3. Let $\mathcal{L}$ be an abstract bi-intuitionistic logic which extends BIL, is preserved under bi-asimulations, $\star$-compact, and has TUP, and let $(\mathcal{M}, w) \in$ Pmod $_{\Theta}$. Then there exists a $(\mathcal{N}, w) \in \operatorname{Pmod}_{\Theta}$ such that $T h_{\mathcal{L}}(\mathcal{M}, w)=T h_{\mathcal{L}}(\mathcal{N}, w)$ and $\mathcal{N}$ is $\mathcal{L}$-saturated.

Proof. It follows from invariance of $\mathcal{L}$ under bi-asimulations and Lemma 7 that $T h_{\mathcal{L}}(\mathcal{M}, w)=T h_{\mathcal{L}}\left(\mathcal{M}_{w}^{u n}, w\right)$. Next, applying Proposition $2 \omega$ times, we form an $\mathcal{L}$-elementary chain of submodels

$$
\mathcal{M}_{w}^{u n}=\mathcal{N}_{1} \preccurlyeq \mathcal{L} \ldots \preccurlyeq \mathcal{L} \mathcal{N}_{n} \preccurlyeq \mathcal{L} \ldots
$$

such that for every $i>0$, we have $\left(\mathcal{N}_{i}, w\right) \in \operatorname{Str}_{\mathcal{L}}(\Theta)$ and $\mathcal{N}_{i+1}$ realizes every $\mathcal{L}$-type of $\mathcal{N}_{i}$. We then set $\mathcal{N}:=\bigcup_{i>0} \mathcal{N}_{i}$ so that $(\mathcal{N}, w) \in \operatorname{Str}_{\mathcal{L}}(\Theta)$. Since $\mathcal{L}$ has TUP, we have then $\mathcal{N}_{i} \preccurlyeq \mathcal{L} \mathcal{N}$ for every $i>0$. In particular, we have $\mathcal{M}_{w}^{u n}=\mathcal{N}_{1} \preccurlyeq \mathcal{L} \mathcal{N}$, so that $T h_{\mathcal{L}}(\mathcal{M}, w)=T h_{\mathcal{L}}(\mathcal{N}, w)$ follows. It remains to show the $\mathcal{L}$-saturation of $\mathcal{N}$, that is to say, that $\mathcal{N}$ realizes all of its $\mathcal{L}$-types. Since we have two sorts of types in $\mathcal{L}$, we have to consider two possible cases.

Case 1. For some $v \in U$ the sets $\Gamma, \Delta \subseteq L(\Theta)$ are such that $(\Gamma, \Delta)$ is a successor $\mathcal{L}$-type of $(\mathcal{N}, v)$. Then, by Corollary 1,1 , for all $\Gamma^{\prime} \Subset \Gamma$ and $\Delta^{\prime} \Subset \Delta$, we have $\bigwedge \Gamma^{\prime} \rightarrow \bigvee \Delta^{\prime} \in T h_{\mathcal{L}}(\mathcal{N}, v)$. But then, we can choose a $j>0$ such that $\mathcal{N}_{j}$ is the first model in the chain for which we have $v \in U_{j}$. Since we also have $\mathcal{N}_{j} \preccurlyeq \mathcal{L} \mathcal{N}$, it follows that $T h_{\mathcal{L}}\left(\mathcal{N}_{j}, v\right)=T h_{\mathcal{L}}(\mathcal{N}, v)$ and thus, for all $\Gamma^{\prime} \Subset \Gamma$ and $\Delta^{\prime} \Subset \Delta$, we have $\bigwedge \Gamma^{\prime} \rightarrow \bigvee \Delta^{\prime} \in T h_{\mathcal{L}}\left(\mathcal{N}_{j}, v\right)$. But then, by Corollary 11,1, $(\Gamma, \Delta)$ must be a successor $\mathcal{L}$-type of $\left(\mathcal{N}_{j}, v\right)$, and, therefore, $(\Gamma, \Delta)$ must be realized in $\mathcal{N}_{j+1}$. But, since we also have $\mathcal{N}_{j+1} \preccurlyeq \mathcal{L} \mathcal{N}$, it follows from Lemma 2 1, that $(\Gamma, \Delta)$ also must be realized in $\mathcal{N}$ itself.

Case 2, where we assume that our $\mathcal{L}$-type is a predecessor $\mathcal{L}$-type, is solved in the same manner. The only difference from Case 1 is that we need to apply Corollary 12 in place of Corollary 11.

We are now in a position to prove Theorem 1. Indeed, assume the hypothesis of the theorem, and assume, for contradiction, that $\mathcal{L} \not \equiv \mathcal{L}^{\prime}$. By Proposition1, there must be a $\phi \in L\left(\Theta_{\phi}\right)$ and $\left(\mathcal{M}_{1}, w_{1}\right),\left(\mathcal{M}_{2}, w_{2}\right) \in \operatorname{Pmod}_{\Theta_{\phi}}$ such that $T h_{B I L}^{+}\left(\mathcal{M}_{1}, w_{1}\right) \subseteq T h_{B I L}^{+}\left(\mathcal{M}_{2}, w_{2}\right)$ while $\mathcal{M}_{1}, w_{1} \models_{\mathcal{L}} \phi$ and $\mathcal{M}_{2}, w_{2} \not \models_{\mathcal{L}} \phi$. By Corollary 3 take $\mathcal{L}$-saturated $\Theta_{\phi}$-models $\mathcal{N}_{1}$ and $\mathcal{N}_{2}$ such that $T h_{\mathcal{L}}\left(\mathcal{M}_{i}, w_{i}\right)=T h_{\mathcal{L}}\left(\mathcal{N}_{i}, w_{i}\right)$ for $i \in\{1,2\}$. We will have then, of course, that $\mathcal{N}_{1}, w_{1} \models_{\mathcal{L}} \phi$, but $\mathcal{N}_{2}, w_{2} \nvdash_{\mathcal{L}} \phi$. On the other hand, we will still have

$$
T h_{\mathcal{L}^{\prime}}^{+}\left(\mathcal{N}_{1}, w_{1}\right) \subseteq T h_{\mathcal{L}^{\prime}}^{+}\left(\mathcal{N}_{2}, w_{2}\right),
$$

whence, by Corollary 2 there must be a bi-asimulation $A$ from $\left(\mathcal{N}_{1}, w_{1}\right)$ to $\left(\mathcal{N}_{2}, w_{2}\right)$, but then, since $\mathcal{L}$ is preserved under bi-asimulations, we must also have

$$
T h_{\mathcal{L}}^{+}\left(\mathcal{N}_{1}, w_{1}\right) \subseteq T h_{\mathcal{L}}^{+}\left(\mathcal{N}_{2}, w_{2}\right) .
$$

Now, since $\phi \in T h_{\mathcal{L}}^{+}\left(\mathcal{N}_{1}, w_{1}\right)$, we can see that we must also have $\phi \in T h_{\mathcal{L}}^{+}\left(\mathcal{N}_{2}, w_{2}\right)$, so that $\mathcal{N}_{2}, w_{2} \models_{\mathcal{L}} \phi$, which is a contradiction.

\section{Conclusion}

In this paper we have extended the methods of [3] to the case of bi-intuitionistic logic, showing bi-intuitionistic propostional logic to be the strongest abstract logic satisfying the Tarski Union Property, a variation of compactness and preservation under bi-asimulations. This extension is rather non-trivial given the complexity of the unravelling construction in the bi-intuitionistic context and the added complexity in building 
the desired saturated structures required for the proof to go through. It would be interesting to find other Lindström characterizations of bi-intuitionistic propositional logic (perhaps finding some natural property that can replace the Tarski Union Property) but we will leave this task for another occasion, as we do not know how to proceed.

\section{REFERENCES}

[1] G. Badia. Bi-simulating in bi-intuitionistic logic. Studia Logica, 104 (5): 1037-1050 (2016).

[2] G. Badia. A remark on Maksimova's variable separation property in super-bi-intuitionistic logics. Australasian Journal of Logic, 14 (1): 46-53 (2017).

[3] G. Badia and G. Olkhovikov. A Lindström theorem for intuitionistic propositional logic. Notre Dame Journal of Formal Logic, 61 (1): 11-30 (2020).

[4] J. Barwise. Axioms for abstract model theory. Annals of Mathematical Logic 7: 221-265 (1974).

[5] J. Barwise, S. Feferman, eds.. Model-Theoretic Logics, Springer-Verlag (1985).

[6] P. Blackburn, M. de Rijke, Y. Venema. Modal Logic, CUP (2001).

[7] A. Chagrov and M. Zakharyaschev. Modal Logic, Clarendon Press (1997).

[8] M. De Rijke. A Lindström Theorem for Modal Logic. In: Modal Logic and Process Algebra. Lecture Notes 53, CSLI Publications, Stanford (1995).

[9] S. Enqvist. A General Lindström Theorem for Some Normal Modal Logics. Logica Universalis, 7(2): 233-264 (2013)

[10] S. Feferman. Two notes on abstract model theory. I. Properties invariant on the range of definable relations between structures. Fundamenta Mathematicae, 82: 153-165 (1974) .

[11] D. Gabbay and L. Maksimova. Interpolation and Definability: Modal and Intuitionistic Logics, OUP (2005).

[12] M. García-Matos and J. Väänänen. Abstract Model Theory as a Framework for Universal Logic. In: Logica Universalis: Towards a General Theory of Logic, Jean-Yves Beziau (ed.), Birkhäuser (2005).

[13] R. Goré and L. Postniece. Combining Derivations and Refutations for Cut-free Completeness in Bi-intuitionistic Logic. Journal of Logic and Computation 20 (1): 233-260 (2010) .

[14] J. de Groot and D. Pattinson. Hennessy-Milner Properties for (Modal) Bi-intuitionistic Logic. In: Iemhoff R., Moortgat M., de Queiroz R. (eds) Logic, Language, Information, and Computation. WoLLIC 2019. Lecture Notes in Computer Science, vol 11541. Springer, Berlin, Heidelberg (2019).

[15] T. Kowalski and H. Ono. Analytic cut and interpolation for bi-intuitionistic logic. Review of Symbolic Logic, 10 (2): 259-283 (2017).

[16] P. Lindström. On extensions of elementary logic. Theoria 35: 1-11 (1969).

[17] P. Lindström. A Characterization of Elementary Logic. In: Modality, Morality, and Other Problems of Sense and Nonsense, pp. 189-191. Lund: Gleerups Bokforlag (1973).

[18] P. Lindström. On Characterizing Elementary Logic. In S. Stenlund (ed.), Logical Theory and Semantic Analysis, Synthese Library vol. 63, pp. 129-146. Dordrecht: D. Reidel (1974).

[19] P. Lindström. Omitting Uncountable Types and Extensions of Elementary Logic. Theoria, 44: 152-156 (1978).

[20] G. K. Olkhovikov. Model-theoretic Characterization of Intuitionistic Propositional Formulas. The Review of Symbolic Logic, 6 (2): 348-365 (2013).

[21] G. K. Olkhovikov. On generalized Van-Benthem-type characterizations. Annals of Pure and Applied Logic , 168 (9): 1643-1691 (2017).

[22] R. Piro and M. Otto. A Lindström Characterisation of the Guarded Fragment and of Modal Logic With a Global Modality. In: Advances in Modal Logic 7, AiML 2008, C. Areces and R. Goldblatt (eds), pp. 273-288.

[23] L. Pinto, T. Uustalu, A proof-theoretic study of bi-intuitionistic propositional sequent calculus. Journal of Logic and Computation, 28 (1): 165-202 (2018).

[24] D. Skurt. Bi-intuitionistic implication structures. Journal of Applied Non-Classical Logics, 28(1), 20-34 (2018).

[25] L. Tranchini, Natural deduction for bi-intuitionistic logic. Journal of Applied Logic 25: S72-S96 (2017).

[26] G. Restall. Extending Intuitionistic Logic with Subtraction (1997). URL: http://consequently.org/writing/ 
[27] C. Rauszer. A formalization of the propositional calculus in H-B logic. Studia Logica, 33 : 23-34 (1974).

[28] C. Rauszer. Model Theory for an Extension of Intuitionistic Logic. Studia Logica, 36: 73-87 (1977).

[29] C. Rauszer. Applications of Kripke Models to Heyting-Brouwer Logic. Studia Logica 36 (1/2): 61-71 (1977).

[30] J. van Benthem. A new modal Lindström theorem. Logica Universalis 1 (1):125-138 (2007).

Department of Philosophy I, Ruhr University Bochum, Bochum, Germany

Department of Philosophy, Ural Federal University, Ekaterinburg, Russia

Email address: grigory.olkhovikov@rub.de, grigory.olkhovikov@gmail.com

Institute of Philosophy and Scientific Method, Johannes Kepler University, Linz, Austria

School of Historical and Philosophical Inquiry, University of Queensland, Brisbane, Australia

Email address: guillebadia89@gmail.com 